\title{
Generalized evolution equations for nonlinear surface gravity waves over two-dimensional topography
}

\author{
By T. T. JANSSEN ${ }^{1}$, T. H. C. HERBER ${ }^{2}$ AND J. A. BATTJES 1 \\ ${ }^{1}$ Environmental Fluid Mechanics, Faculty of Civil Engineering and Geosciences, Delft University of \\ Technology, Stevinweg 1, 2628 CN, Delft, The Netherlands \\ ${ }^{2}$ Department of Oceanography, Naval Postgraduate School, Monterey, CA 93943, USA
}

(Received 4 July 2005 and in revised form 22 September 2005)

Evolution equations are derived for weakly nonlinear, multi-frequency and directional surface gravity waves propagating from deep to shallow water over weakly twodimensional bottom topography. A uniform transition from cubic resonances in deepintermediate water (Stokes regime) to quadratic near resonances in shallow water (Boussinesq regime) is obtained by extending the ordered solution to include additional higher-order terms for the bound wave components. The model assumes a leading-order, alongshore-uniform bottom with a two-dimensional depth perturbation that is incorporated through a Taylor series expansion of the bottom boundary condition. Numerical implementations of the model and comparisons to experimental data are presented that demonstrate the model's ability to describe: (i) cubic wavewave interactions in deep-intermediate water depth; (ii) harmonic generation over a one-dimensional submerged obstacle; (iii) harmonic generation over two-dimensional topography.

\section{Introduction}

As ocean waves advance from deep water into shallow coastal areas and onto beaches they transform owing primarily to refraction and nonlinear wave interactions, the latter dominated by near-resonant quadratic interactions involving triplets of waves. Sum interactions transform near-symmetrical waves to the characteristic skewed, pitched-forward shapes of waves observed on beaches (e.g. Elgar \& Guza 1985) and cause the formation of multi-crest wave trains behind submerged obstacles (e.g. Johnson, Fuchs \& Morison 1951; Byrne 1969). Difference interactions induce radiation of long-wave motion in the nearshore region, generally referred to by the collective name 'surf beat', coined by its pioneering observer Munk (1949). These effects are recognized as major factors in the study of nearshore morphological evolution (e.g. Roelvink \& Stive 1989; Hoefel \& Elgar 2003) and are of paramount importance in the design of coastal structures and harbours.

In deep-intermediate water, frequency dispersion precludes resonances in the quadratic interactions (the lowest order nonlinear interactions); instead, the nonlinear wave field evolution is dominated by cubic or quartet interactions (e.g. Phillips $1977, \S 3.8)$. These interactions are an order higher in the nonlinearity parameter $\epsilon \ll 1$ than quadratic interactions, and thus affect wave evolution over distances that are $O\left(\epsilon^{-1}\right)$ longer. The Zakharov equation (Zakharov 1968) and the deterministic 
expressions in Hasselmann (1962) constitute evolution equations incorporating the effects of cubic interactions without limitations on spectral width. Stiassnie \& Shemer (1984) re-derived Zakharov's work for finite depth and Herterich \& Hasselmann (1980) corrected a typographical error affecting the finite depth expressions in Hasselmann (1962). The celebrated cubic Schrödinger equation (CSE) (e.g. Zakharov 1968; Hasimoto \& Ono 1972; Yuen \& Lake 1975) and the Dysthe equation (Dysthe 1979) are special cases of these general formulations when a perturbation in spectral width is applied. Depth variability was incorporated in the derivations of e.g. Chu \& Mei (1970), Djordjević \& Redekopp (1978) and Liu \& Dingemans (1989), but apart from the one-dimensional simulations in Dingemans et al. (1991), few numerical implementations have been reported. Suh, Dalrymple \& Kirby (1990) derived an angular spectrum model for Stokes waves over weakly two-dimensional topography and compared model simulations to laboratory observations. Invariably these models, with cubic nonlinearities as the dominant nonlinear physics, incorporate quadratic interactions as bound second-order corrections to the primary waves, rendering them incompatible with shallow water where quadratic interactions approach resonance (e.g. Bryant 1974).

Following Armstrong et al. (1962) and Bretherton (1964), evolution equations based on Boussinesq theory were developed to describe the effects of quadratic nearresonances in uniform depth (e.g. Mei \& Ünlüata 1972; Bryant 1973), over undulating topography (Lau \& Barcilon 1972) and over arbitrary but mildly varying depth (Freilich \& Guza 1984). Keller (1988) showed that similar equations are in fact derivable from fully dispersive theory. Many models have emerged since then, either based on Boussinesq theory (e.g. Madsen \& Sørensen 1993; Herbers \& Burton 1997) or fully dispersive theory, suitable for unidirectional waves over one-dimensional topography (e.g. Agnon et al. 1993), small-angle models for two-dimensional topography (e.g. Kaihatu \& Kirby 1995; Tang \& Ouellet 1997) and models for multi-directional wave propagation over alongshore-uniform topography (e.g. Sheremet 1996; Eldeberky \& Madsen 1999). In general, the models based on fully dispersive theory include full dispersion in the linear terms and the nonlinear interaction coefficient but retain the premise of near-resonance. The latter restriction was removed by Bredmose et al. (2002) who apply suitable boundary conditions on a general solution to the Laplace equation in the form of infinite expansions of trigonometric functions (see also Madsen \& Schäffer 1998; Rayleigh 1876) for unidirectional waves over onedimensional topography. These models include quadratic near-resonances as the dominant nonlinear physics but invariably lack the ability to model cubic near resonances in deep-intermediate depth, which implicitly restricts them to relatively shallow water where the assumption of dominant quadratic interactions is warranted.

The aim of the present work is to derive generalized evolution equations suitable for surface wave propagation over two-dimensional topography including the transition from cubic wave-wave interactions in deep-intermediate water (Stokes regime) to quadratic interactions in shallow water (Boussinesq regime) in a consistent and unified manner. Our derivation is along the lines of Chu \& Mei (1970), generalized to a multi-frequency and multi-directional wave field utilizing an angular spectrum decomposition of the wave field (e.g. Dalrymple et al. 1989), and extended to higher order in the bound wave components to support the transition to shallow water. The effects of topographical features on the wave propagation are included through a scattering mechanism (e.g. Suh et al. 1990). The resulting model does not break down in shallow water (where the Stokes number is $O(1)$ ), as is typical for models based on Stokes-type expansions, but instead reduces to a Boussinesq-type approximation. 
In $\S 2$ we define the wave field and bottom decomposition, introduce notational conventions and a general treatment of secular forcing terms in the ordered boundary value problem. The finite-depth solutions are given in $\S 3$ and extended to shallow water in $\S 4$. Based on these results, a uniformly valid evolution equation is presented in $\S 5$ and the equivalence of asymptotic limits with established expressions from the literature is addressed. Model simulations are compared to laboratory observations in $\S 6$, followed by a summary in $\S 7$.

\section{General theory}

The starting point of our derivation is the governing set of equations for irrotational flow of an incompressible, inviscid fluid:

$$
\begin{aligned}
& \nabla^{2} \Phi+\Phi_{z z}=0, \quad \forall z \in \mathscr{D}, \\
& \Phi_{t t}+g \Phi_{z}+\left[\partial_{t}+\frac{1}{2} \nabla \Phi \cdot \nabla+\frac{1}{2} \Phi_{z} \partial_{z}\right]\left\{|\nabla \Phi|^{2}+\left(\Phi_{z}\right)^{2}\right\}=0, \quad z=\eta(x, y, t), \\
& \Phi_{z}+\nabla h \cdot \nabla \Phi=0, \quad z=-h(x, y), \\
& g \eta+\Phi_{t}+\frac{1}{2}\left(|\nabla \Phi|^{2}+\left(\Phi_{z}\right)^{2}\right)=0 . \quad z=\eta(x, y, t) .
\end{aligned}
$$

Here $\Phi$ is a velocity potential function, $\eta$ is the surface elevation and $g$ denotes gravitational acceleration. We use a Cartesian frame of reference with its origin at the undisturbed free surface of the fluid: $x, y$ denote the two horizontal coordinates and $z$ corresponds to the vertical coordinate, positive pointing upward. The operator $\nabla \equiv\left\langle\partial_{x}, \partial_{y}\right\rangle$, where $\partial_{x}$ is a shorthand for partial differentiation with respect to the subscripted variable. The Laplace equation (2.1a) follows from continuity. The freesurface boundary condition $(2.1 \mathrm{~b})$ combines the kinematic and dynamic conditions while assuming a constant atmospheric pressure (see e.g. Phillips 1977, $\$ 3.1$ or Dingemans 1997, §1.3). Impermeability of the bottom is expressed by $(2.1 c)$, and the surface elevation is related to the velocity potential by the dynamic free-surface boundary condition (2.1d).

\subsection{Decomposition for weakly two-dimensional topography}

We consider nonlinear surface waves propagating in finite water depth over slowly varying topography with weakly two-dimensional features. The topography is considered one-dimensional to leading order with a superposed two-dimensional perturbation, written as

$$
h(x)=\bar{h}(x)-\widetilde{h}(x) .
$$

Here $\bar{h}$ represents the lateral average of $h$ and $\widetilde{h}$ the (two-dimensional) residue. Without loss of generality, we let the $x$ - and $y$-axes coincide with the principal and lateral direction respectively, and since we are particularly interested in the description of waves propagating from the deep open ocean into a shallow coastal area, we refer to the principal direction as the cross-shore direction and the lateral direction as the alongshore direction.

The magnitude of the lateral depth variations, $\widetilde{h}$, is characterized by the small parameter $\gamma$ defined as

$$
O(\gamma)=O\left(\frac{\widetilde{h}_{0}}{\bar{h}_{0}}\right) \ll 1
$$

where $\widetilde{h}_{0}$ is a characteristic amplitude of $\widetilde{h}$ and $\bar{h}_{0}$ a reference depth. 
The nonlinearity of the wave field is governed by the parameter $O(\epsilon)=O\left(a_{0} / L_{v}\right) \ll 1$, where $a_{0}$ and $L_{v}$ denote a characteristic amplitude of the surface elevation and a representative vertical length scale of the wave motion respectively. Since we consider wave propagation from deep to shallow water we choose a generally applicable vertical length scale $L_{v}=\mu / k_{0}$, where $k_{0}$ is a representative wavenumber of the wave motion and $\mu=\tanh k_{0} \bar{h}_{0}$, which can be considered a generalized dispersion parameter varying from $O(1)$ in deep-intermediate water to $O\left(k_{0} \bar{h}_{0}\right)$ in shallow water (see also Beji 1995; Kirby 1998).

The depth variations are assumed small over distances $O\left(k_{0}^{-1}\right)$, made explicit by the parameter $\beta$, where

$$
O(\beta)=O\left(\frac{\partial_{x} \bar{h}}{k_{0} \bar{h}}\right)=O\left(\frac{|\nabla \widetilde{h}|}{k_{0} \bar{h}}\right) \ll 1 .
$$

We set the relative magnitudes of the relevant small parameters to $O(\beta)=O\left(\gamma^{2}\right)=$ $O\left(\epsilon^{2} / \mu^{2}\right)$ and define multiple scales (see e.g. Chu \& Mei 1970; Liu \& Dingemans 1989; Suh et al. 1990):

$$
\left\langle t_{n}, x_{n}, y_{n}\right\rangle=\left(\frac{\epsilon}{\mu}\right)^{n}\langle t, x, y\rangle, \quad n=\{1,2\} .
$$

To make the ordering of the bottom perturbation explicit in the derivation we write

$$
\widetilde{h}=\gamma \widehat{h}, \quad \nabla \widetilde{h}=\gamma^{2} \nabla_{1} \widehat{h}
$$

where $\widehat{h} \sim O(1)$ and $\nabla_{1} \equiv\left\langle\partial_{x_{1}}, \partial_{y_{1}}\right\rangle$.

In the present scaling the bottom slope is $O\left(\epsilon^{2} / \mu^{2}\right)$, affecting wave evolution at $O\left(\epsilon^{2}\right)$ in deep-intermediate water $(O(\mu) \sim O(1))$ and $O(\epsilon)$ in shallow water $\left(O\left(\mu^{2}\right) \sim\right.$ $O(\epsilon)$ ). This approximation is well-suited to typical coastal bathymetry with a sloping beach extending onto a relatively flat continental shelf. Moreover, the relative magnitude of the lateral depth variations, $\widetilde{h}$, is introduced at lower order, $O(\epsilon / \mu)$, to accurately resolve wave propagation over shallow submerged bathymetric features such as banks and shoals that are common in coastal areas. The scaling of (2.2)-(2.4) further implies that the characteristic length scale of the two-dimensional topography is long, $O(\mu / \epsilon)$, compared with the surface wavelength and thus the back-scattering of waves (induced by bottom undulations of about half the surface wavelength) is neglected in the present approximation, consistent with estimates of wave scattering from natural continental shelf topography presented in earlier studies (e.g. Ardhuin \& Herbers 2002).

Following Chu \& Mei (1970), the set (2.1) is solved by applying a perturbation expansion of the surface elevation and velocity potential. In order to capture both the leading-order effects of cubic resonances in deep-intermediate water and quadratic resonances in shallow water we explicitly evaluate the bound-wave components induced by quadratic wave-wave interactions:

$$
\left[\begin{array}{l}
\Phi \\
\eta
\end{array}\right]=\sum_{n=1}^{\infty} \frac{\epsilon^{n}}{\mu^{n-1}}\left\{\left[\begin{array}{l}
\Phi^{(n, 0)} \\
\eta^{(n, 0)}
\end{array}\right]+\left[\begin{array}{l}
\Phi^{(n, 1)} \\
\eta^{(n, 1)}
\end{array}\right]+\frac{\epsilon}{\mu^{2}}\left[\begin{array}{l}
\Phi^{(n+1,2)} \\
\eta^{(n+1,2)}
\end{array}\right]\right\}+\mathrm{HBC}
$$

The $(n, 0)$ components correspond to wave-driven mean flow, $(n, 1)$ to free waves, and $(n+1,2)$ to quadratically coupled or bound waves. Anticipating the near-resonance of quadratic nonlinear forcing terms in shallow water, these are scaled with $\epsilon / \mu^{2}$ $\left(=k_{0} a_{0} /\left(\tanh k_{0} \bar{h}_{0}\right)^{3}\right)$ which has the appropriate deep- and shallow-water asymptotes of wave steepness, $a_{0} k_{0}$, and the Stokes number, $a_{0} /\left(k_{0}^{2} \bar{h}_{0}^{3}\right)$ (Stokes 1847$)$, respectively. 
The HBC in (2.6) denotes higher-order bound components (involving cubic and higher-order, non-resonant interactions) that will not be considered in the present analysis (see also Shemer et al. 2001).

The premise of a leading-order, alongshore-uniform topography supports a frequency $(\omega)$-alongshore wavenumber $(\lambda)$ or angular-spectrum decomposition (see e.g. Suh et al. 1990). Accordingly we write the primary wave field (lowest-order free waves) as a summation of modulated plane waves propagating at discrete angles in the positive $x$-direction:

$$
\left[\begin{array}{l}
\Phi^{(1,1)} \\
\eta^{(1,1)}
\end{array}\right]=\sum_{\substack{p, q=-\infty \\
p \neq 0}}^{\infty}\left[\begin{array}{l}
\widetilde{\phi}_{q, p}^{(1,1)} \\
\widetilde{\zeta}_{q, p}^{(1,1)}
\end{array}\right] \exp \left[\mathrm{i}\left(\lambda_{q} y-\omega_{p} t\right)\right]=\sum_{v}\left[\begin{array}{l}
\phi_{v}^{(1,1)} \\
\zeta_{v}^{(1,1)}
\end{array}\right] E_{v}
$$

Here $\lambda_{q}=q \Delta \lambda$ and $\omega_{p}=p \Delta \omega$ in which $\Delta \lambda$ and $\Delta \omega$ represent the discrete alongshore wavenumber and angular frequency spacing respectively. The summation over $\boldsymbol{v}=$ $\langle q, p\rangle$ is a shorthand for the summation over all combinations of the discrete frequencies and alongshore wavenumbers. We exclude zero-frequency components $(p=0)$ since these are part of the mean flow and are treated separately (Appendix C). The $\left\{\phi_{v}^{(1,1)}, \zeta_{v}^{(1,1)}\right\}$ are slowly varying amplitudes while the rapid phase variations are incorporated in $E_{v}$, given as

$$
E_{v}=\exp \left[\mathrm{i}\left(\psi_{v}(x)+\lambda_{q} y-\omega_{p} t\right)\right], \quad \psi_{v}(x)=\int^{x} \chi_{v}\left(x^{\prime}\right) \mathrm{d} x^{\prime} .
$$

Here $\varkappa_{v}=\operatorname{sgn}(p) \sqrt{k_{p}^{2}-\lambda_{q}^{2}}$, with sgn denoting the signum function, and $k_{p}$ is related to the angular frequency, $\omega_{p}$, through the lowest-order dispersion relation. Since $\Phi$ and $\eta$ are real functions we have $\phi_{v}^{(1,1)}=\phi_{-v}^{(1,1) *}$ and $\zeta_{v}^{(1,1)}=\zeta_{-v}^{(1,1)^{*}}$, where * denotes the complex conjugate.

In the present work we consider only propagating modes. We omit exponentially decaying (evanescent) modes, such as refractively trapped waves for which $\left|\lambda_{q}\right|>k_{p}$, and vertical eigenmodes. Such non-propagating modes may e.g. be excited around bathymetric features (refractive trapping) or on steep slopes and at domain boundaries; they can be important locally, but are confined to the near field (typically a few wavelengths) of their generation source. Away from such regions, at distances much greater than $O\left(\chi_{v}^{-1}\right)$, the wave field is accurately represented by the propagating modes (Stamnes 1986). The complete solution to the linearized problem and further discussion on the validity of the present approximation is given in $\S 19.1$ of Stamnes (1986).

Using the spectral decomposition (2.7), the total wave field (2.6) can be expressed as

$$
\left[\begin{array}{l}
\Phi \\
\eta
\end{array}\right]=\sum_{n=1}^{\infty} \frac{\epsilon^{n}}{\mu^{n-1}}\left\{\left[\begin{array}{l}
\Phi^{(n, 0)} \\
\eta^{(n, 0)}
\end{array}\right]+\sum_{\boldsymbol{v}_{1}}\left[\begin{array}{l}
\phi_{1}^{(n, 1)} \\
\zeta_{1}^{(n, 1)}
\end{array}\right] E_{1}+\frac{\epsilon}{\mu^{2}} \sum_{\boldsymbol{v}_{1}, \boldsymbol{v}_{2}}\left[\begin{array}{l}
\phi_{12}^{(n+1,2)} \\
\zeta_{12}^{(n+1,2)}
\end{array}\right] E_{12}\right\}+\text { HBC. }
$$

For notational convenience, we write $E_{12 \ldots n}=E_{1} E_{2} \ldots E_{n}$ and apply numerical subscripts to identify individual wave components, for example $\phi_{1}^{(n, 1)}$ is a shorthand for $\phi_{v_{1}}^{(n, 1)}$ denoting the velocity potential function for free wave component $\boldsymbol{v}_{1}$. Double subscripts without separator always refer to the corresponding bound wave component; accordingly, $\phi_{12}^{(2,2)}$ denotes the bound wave component resulting from the quadratic interaction of $\phi_{1}^{(1,1)}$ and $\phi_{2}^{(1,1)}$.

The lateral variability of the wave field is captured by the decomposition in angular components so that the modulation of the amplitudes due to wave-wave, wavecurrent and wave-bottom interactions, as well as the effects of non-stationarity, take 
place on slow scales in $x$ and $t$ alone:

$$
\left[\begin{array}{c}
\phi_{a_{m}}^{(n, m)} \\
\zeta_{a_{m}}^{(n, m)}
\end{array}\right]=\left[\begin{array}{c}
\phi_{a_{m}}^{(n, m)}\left(z, x_{1}, t_{1}, x_{2}, t_{2}\right) \\
\zeta_{a_{m}}^{(n, m)}\left(x_{1}, t_{1}, x_{2}, t_{2}\right)
\end{array}\right], \quad m=1,2, \quad n \geqslant m,
$$

where the subscript $a_{m}=1$ for $m=1$ and $a_{m}=12$ for $m=2$, corresponding to components $\boldsymbol{v}_{1}$ and $\left\{\boldsymbol{v}_{1}, \boldsymbol{v}_{2}\right\}$ respectively.

\subsection{The ordered solution}

Upon substituting the wave field decomposition in the Laplace equation $(2.1 a)$, the bottom boundary condition $(2.1 c)$ and the free-surface boundary condition $(2.1 d)$, these can be written in the form (see e.g. Chu \& Mei 1970; Liu \& Dingemans 1989)

$$
\begin{aligned}
\left(\mathrm{d}_{z}^{2}-k_{a_{m}}^{2}\right) \phi_{a_{m}}^{(n, m)} & =R_{a_{m}}^{(n, m)}, & & \forall z \in \mathscr{D}, \\
\mathrm{d}_{z} \phi_{a_{m}}^{(n, m)} & =F_{a_{m}}^{(n, m)}, & z & =-\bar{h}, \\
\zeta_{a_{m}}^{(n, m)} & =\frac{1}{g}\left[\mathrm{i} \omega_{a_{m}} \phi_{a_{m}}^{(n, m)}-H_{a_{m}}^{(n, m)}\right], & & z=0,
\end{aligned}
$$

where $\mathrm{d}_{z}$ denotes differentiation with respect to $z, n \geqslant m$ and the forcing terms $R_{a_{m}}^{(n, m)}$, $F_{a_{m}}^{(n, m)}$ and $H_{a_{m}}^{(n, m)}$ on the right-hand side of (2.11) depend on lower-order results and are specified below as needed.

A general solution to $(2.11 a)$ and the condition $(2.11 b)$ can be found through variation of parameters (e.g. Chu \& Mei 1970):

$$
\phi_{a_{m}}^{(n, m)}=-\frac{\mathrm{Ch} Q_{a_{m}}}{k_{a_{m}}^{2}}\left[I_{1}^{(n, m)}\left(Q_{a_{m}}\right)+\mathscr{M}_{a_{m}}^{(n, m)}\right]+\frac{\operatorname{Sh} Q_{a_{m}}}{k_{a_{m}}^{2}}\left[I_{2}^{(n, m)}\left(Q_{a_{m}}\right)+k_{a_{m}} F_{a_{m}}^{(n, m)}\right]
$$

where $\mathscr{M}_{a_{m}}^{(n, m)}$ is an integration constant,

$$
Q_{a_{m}}=k_{a_{m}}(\bar{h}+z), \quad \operatorname{Ch}\{\}=\cosh \{\}, \quad \operatorname{Sh}\{\}=\sinh \{\}
$$

and

$$
\begin{aligned}
& I_{1}^{(n, m)}\left(Q_{a_{m}}\right)=\int_{0}^{Q_{a_{m}}} R_{a_{m}}^{(n, m)} \operatorname{Sh} Q_{a_{m}}^{\prime} \mathrm{d} Q_{a_{m}}^{\prime}, \\
& I_{2}^{(n, m)}\left(Q_{a_{m}}\right)=\int_{0}^{Q_{a_{m}}} R_{a_{m}}^{(n, m)} \mathrm{Ch} Q_{a_{m}}^{\prime} \mathrm{d} Q_{a_{m}}^{\prime} .
\end{aligned}
$$

Insertion of (2.12) in the combined free-surface boundary condition $(2.1 b)$ yields

$$
\Delta_{a_{m}}\left[I_{1}^{(n, m)}\left(q_{a_{m}}\right)+\mathscr{M}_{a_{m}}^{(n, m)}\right]-\Gamma_{a_{m}}\left[I_{2}^{(n, m)}\left(q_{a_{m}}\right)+k_{a_{m}} F_{a_{m}}^{(n, m)}\right]=-S_{a_{m}}^{(n, m)} \frac{k_{a_{m}}}{g \operatorname{Sh} q_{a_{m}}}
$$

where the forcing term $S_{a_{m}}^{(n, m)}$ is the result of the perturbation expansion for the wave field, the expansion of the coordinates in multiple scales and the Taylor expansion of $(2.1 b)$ around $z=0$. We have used the shorthand notation

$$
\Delta_{a_{m}}=1-\frac{\omega_{a_{m}}^{2}}{g k_{a_{m}} T_{a_{m}}}, \quad \Gamma_{a_{m}}=\frac{1}{T_{a_{m}}}-\frac{\omega_{a_{m}}^{2}}{g k_{a_{m}}}, \quad T_{a_{m}}=\tanh q_{a_{m}}, \quad q_{a_{m}}=k_{a_{m}} \bar{h},
$$

where $\Delta_{a_{m}}$ can be interpreted as a measure of resonance mismatch.

Note from (2.16) that $m=1$ is a special case in the sense that $\Delta_{a_{1}}$ vanishes (i.e. the forcing is secular) so that (2.16) constitutes a solvability condition on the lower-order wave field, but with $\Delta_{a_{1}}=0$ it leaves the homogeneous part of $\phi_{1}^{(n, 1)}$ (namely $\mathscr{M}_{a_{1}}^{(n, 1)}$ ) 
undetermined (see also Chu \& Mei 1970; Liu \& Dingemans 1989). The derivation of the third-order evolution equations involves operations on $\phi_{1}^{(2,1)}$ and thus the complete solution for $\phi_{1}^{(2,1)}$, including the contribution from $\mathscr{M}_{a_{1}}^{(2,1)}$, is needed to consistently derive such third-order expressions. To this end, Chu \& Mei (1970) asymptotically matched their second-order result to the corresponding deep-water solution, whereas Liu \& Dingemans (1989) derived an evolution equation for the combined first- and second-order free wave $(m=1)$ potential amplitudes. Kennedy \& Kirby (2003) discarded the homogeneous contribution at second order altogether, which in their context is equivalent to the approach of Liu \& Dingemans (1989). In the present context these approaches would either result in ambiguous results or yield modifications of the wave field due to wave-bottom interactions even in deep water, which must be rejected on physical grounds. Therefore, we pursue an explicit derivation of the second-order free wave potential from first principles that includes the contribution from $\mathscr{M}_{a_{1}}^{(2,1)}$ and assures a proper deep-water asymptote (i.e. vanishing contributions of wave-bottom interactions in deep water). To this end, the case $m=1$ (free waves) is considered as the limit of a vanishing mismatch from resonance by writing

$$
\omega_{a_{1}}^{2}=\lim _{\Delta_{a_{1}} \rightarrow 0} g k_{a_{1}} T_{a_{1}}\left(1-\Delta_{a_{1}}\right)
$$

and we expand $\Gamma_{a_{1}}$ and $S_{a_{1}}^{(n, 1)}$ in terms of $\Delta_{a_{1}}$ according to

$$
\begin{aligned}
\Gamma_{a_{1}} & =\Gamma_{a_{1}}^{(0)}+\Delta_{a_{1}} \Gamma_{a_{1}}^{(1)}, \\
S_{a_{1}}^{(n, 1)} & =\left.\sum_{j=0}^{1} \frac{\Delta_{a_{1}}^{j}}{j !} \frac{\mathrm{d}^{j} S_{a_{1}}^{(n, 1)}}{\mathrm{d} \Delta_{a_{1}}^{j}}\right|_{\Delta_{a_{1}}=0}+O\left(\Delta_{a_{1}}^{2}\right) \\
& =S_{a_{1}}^{(n, 1,0)}+\Delta_{a_{1}} S_{a_{1}}^{(n, 1,1)}+O\left(\Delta_{a_{1}}^{2}\right) .
\end{aligned}
$$

Insertion of (2.19) and (2.20) into (2.16) yields

$$
\begin{aligned}
& \mathscr{M}_{a_{1}}^{(n, 1)}=\lim _{\Delta_{a_{1}} \rightarrow 0}\left\{\frac{1}{\Delta_{a_{1}}}\left[\Gamma_{a_{1}}^{(0)}\left(I_{2}^{(n, 1)}\left(q_{a_{1}}\right)+k_{a_{1}} F_{a_{1}}^{(n, 1)}\right)-\frac{k_{a_{1}}}{g \operatorname{Sh} q_{a_{1}}} S_{a_{1}}^{(n, 1,0)}\right]\right. \\
& \left.+\Gamma_{a_{1}}^{(1)}\left(I_{2}^{(n, 1)}\left(q_{a_{1}}\right)+k_{a_{1}} F_{a_{1}}^{(n, 1)}\right)-I_{1}^{(n, 1)}\left(q_{a_{1}}\right)-\frac{k_{a_{1}}}{g \operatorname{Sh} q_{a_{1}}} S_{a_{1}}^{(n, 1,1)}+O\left(\Delta_{a_{1}}\right)\right\}
\end{aligned}
$$

and thus for vanishing $\Delta_{a_{1}}$ we obtain from (2.21)

$$
\mathscr{M}_{a_{1}}^{(n, 1)}=\Gamma_{a_{1}}^{(1)}\left(I_{2}^{(n, 1)}\left(q_{a_{1}}\right)+k_{a_{1}} F_{a_{1}}^{(n, 1)}\right)-I_{1}^{(n, 1)}\left(q_{a_{1}}\right)-\frac{k_{a_{1}}^{2}}{\omega_{a_{1}}^{2}} \frac{S_{a_{1}}^{(n, 1,1)}}{\operatorname{Ch} q_{a_{1}}}
$$

provided that the terms between square brackets in (2.21), multiplied by $\Delta_{a_{1}}^{-1}$, cancel:

$$
\frac{1}{k_{a_{1}}} \int_{0}^{q_{a_{1}}} R_{a_{1}}^{(n, 1)} \mathrm{Ch} Q_{a_{1}}^{\prime} \mathrm{d} Q_{a_{1}}^{\prime}+F_{a_{1}}^{(n, 1)}=\frac{\mathrm{Ch} q_{a_{1}}}{g} S_{a_{1}}^{(n, 1,0)} .
$$

Equation (2.22) unambiguously defines the homogeneous part of the velocity potential while (2.23) is the usual solvability condition. The latter is also found if the limit is not taken explicitly (as done here) but the forcing is considered secular from the outset; however, in that case the contribution given in (2.22) remains undetermined.

For $m=2$ we have $k_{a_{2}}=k_{12}=\left|\boldsymbol{k}_{1}+\boldsymbol{k}_{2}\right|, \omega_{a_{2}}=\omega_{12}=\omega_{1}+\omega_{2}$ and the resonance mismatch is generally $O\left(\mu^{2}\right)$. To make the order explicit we write $\Delta_{a_{2}}=\mu^{2} \widehat{\Delta}_{a_{2}}$ with $\widehat{\Delta}_{a_{2}} \sim O(1)$. The lowest-order forcing problem for $m=2(n=2)$ is inhomogeneous 
only in the free-surface boundary condition involving the forcing term $S_{a_{2}}^{(2,2)}$. Since in (2.9) we expand the bound waves as $O\left(\epsilon^{2} / \mu^{2}\right)$ from the outset rather than $O\left(\epsilon^{2}\right)$ we correspondingly write

$$
\mathscr{M}_{a_{2}}^{(2,2)}=-\frac{k_{a_{2}}}{g T_{a_{2}} \widehat{\Delta}_{a_{2}}} \frac{S_{a_{2}}^{(2,2)}}{\operatorname{Ch} q_{a_{2}}} .
$$

In the present analysis, quadratic bound wave contributions of higher than second order $(n>2)$ are considered only in the near-resonant shallow-water limit (see §4) where the Stokes number is $O(1)$.

\section{Deep-intermediate water solution}

In deep-intermediate water the representative vertical length scale $L_{v}=k_{0}^{-1}$ and the nonlinearity parameter, $\epsilon$, represents wave steepness. Here $\mu \sim O(1)$ and thus can be omitted, simplifying the analysis to an expansion in a single small parameter, $\epsilon$. Our expression for the second-order free wave potential differs from earlier studies (for the reasons explained in $\S 2.2$ ), leading to modifications of the $O\left(\epsilon^{3}\right)$ evolution equations.

\subsection{First order, $O(\epsilon)$}

For $m=1$ the lowest-order solution renders the boundary value problem (2.11) homogeneous, yielding for the primary waves:

$$
\phi_{1}^{(1,1)}=\varphi_{1}^{(1,1)} f_{1}, \quad f_{1}=\frac{\mathrm{Ch} Q_{1}}{\operatorname{Ch} q_{1}}, \quad \varphi_{1}^{(1,1)}=-\mathrm{i} \frac{g}{\omega_{1}} \zeta_{1}^{(1,1)},
$$

and the dispersion relation

$$
\omega_{1}^{2}=g k_{1} T_{1}
$$

where $\varphi_{a_{m}}^{(n, m)}=\left.\phi_{a_{m}}^{(n, m)}\right|_{z=0}$ is the velocity potential amplitude evaluated at the undisturbed surface.

\subsection{Second order, $O\left(\epsilon^{2}\right)$}

At this order, for $m=1$, the set (2.11) is inhomogeneous with secular forcing terms including a quadratic forcing term involving a product of the bottom perturbation, $\widehat{h}$, and the wave potential, $\varphi_{1}^{(1,1)}$, that accounts for the interaction of the wave field with the lateral depth variations:

$$
\begin{aligned}
R_{1}^{(2,1)} & =-\mathrm{i} 2 \varkappa_{1} \phi_{1, x_{1}}^{(1,1)}, \\
S_{1}^{(2,1)} & =2 \mathrm{i} \omega_{1} \varphi_{1, t_{1}}^{(1,1)}, \\
F_{1}^{(2,1)} & =-\frac{k_{1}^{2}}{\operatorname{Ch} q_{1}} \mathscr{G}_{1}\left\{\widehat{h}, \varphi_{2}^{(1,1)}\right\} .
\end{aligned}
$$

The forcing term, $F_{1}^{(2,1)}$, involves the operator

$$
\mathscr{G}_{i}\left\{a, b_{j}\right\}=\mathscr{F}_{i}\left\{a(y) \mathscr{F}^{-1}\left\{b_{j} \exp \left[\mathrm{i} \psi_{j}\right]\right\}\right\} \exp \left[-\mathrm{i} \psi_{i}\right],
$$

where $\mathscr{F}_{i}$ denotes the $i$ th component of the discrete Fourier transform (DFT) with respect to the lateral coordinate, and $\mathscr{F}^{-1}$ denotes the inverse discrete Fourier transform (IDFT). The interaction with the topography is thus modelled as a triad interaction between two wave components with equal frequency and a difference alongshore wavenumber matching that of the (zero-frequency) bottom component, i.e. the triad $\left\{\omega_{1}, \lambda_{1} ; \omega_{1}, \lambda_{2} ; 0, \lambda_{1}-\lambda_{2}\right\}$. This scattering approach, as opposed to more 
conventional approaches for refraction-diffraction modelling, describes wave propagation over two-dimensional topography by a set of one-dimensional (apart from time) transport equations.

From (2.12), using (2.22), we find for the potential function $\phi_{1}^{(2,1)}$

$$
\phi_{1}^{(2,1)}=\left[\mathscr{K}_{1}^{(1,2)}-\mathrm{i}\left(\frac{\varkappa_{1}}{k_{1}^{2}} \varphi_{1, x_{1}}^{(1,1)} Q_{1} T_{Q_{1}}-\mathrm{i} k_{1} \mathscr{G}_{1}\left\{\widehat{h}, \varphi_{2}^{(1,1)}\right\} T_{Q_{1}}\right)\right] \frac{\mathrm{Ch} Q_{1}}{\operatorname{Ch} q_{1}}
$$

where

$$
T_{Q_{1}}=\frac{\operatorname{Sh} Q_{1}}{\operatorname{Ch} Q_{1}}, \quad \mathscr{K}_{1}^{(2,1)}=-\mathrm{i}\left[\frac{\varphi_{1, t_{1}}^{(1,1)}}{\omega_{1}}-\frac{\chi_{1}}{k_{1}^{2}} \varphi_{1, x_{1}}^{(1,1)} q_{1} T_{1}+\mathrm{i} k_{1} T_{1} \mathscr{G}_{1}\left\{\widehat{h}, \varphi_{2}^{(1,1)}\right\}\right] .
$$

Setting $\mathscr{K}_{1}^{(2,1)}=0$ in expression (3.4) yields the corresponding expression in Suh et al. (1990). This implies a non-vanishing contribution of the lateral depth variations to both the second-order velocity potential and the corresponding second-order surface elevation, $\zeta_{1}^{(2,1)}$, which persists in deep water, as can be easily verified. Taking $\mathscr{K}_{1}^{(2,1)}$ into account, as in (3.4), determines $\phi_{1}^{(2,1)}$ unambiguously, such that the topography has a vanishing effect on the velocity potential in deep water. The corresponding surface elevation correction at this order, $\zeta_{1}^{(2,1)}$, vanishes identically, which can be seen from inserting (3.4) into (2.11c).

The solvability condition (2.23) for $n=2$ on the lowest-order wave field is

$$
\mathscr{L}^{(1)}\left\{\varphi_{1}^{(1,1)}\right\}=\xi_{1}^{(2,1, \mathrm{wb})}
$$

where

$$
\mathscr{L}^{(1)}\{\}=\left[\partial_{t_{1}}+V_{1} \partial_{x_{1}}\right], \quad \xi_{1}^{(2,1, \mathrm{wb})}=\mathrm{i} \frac{g}{2 \omega_{1}} k_{1}^{2}\left(1-T_{1}^{2}\right) \mathscr{G}_{1}\left\{\widehat{h}, \varphi_{2}^{(1,1)}\right\},
$$

in which $V_{1}=\left(\left(g \varkappa_{1}\right) /\left(2 \omega_{1} k_{1}\right)\right)\left(T_{1}+q_{1}\left(1-T_{1}^{2}\right)\right)=\left(\varkappa_{1} / k_{1}\right) C_{g, 1}$ is the cross-shore (principal direction) component of the linear group speed vector. The forcing term $\xi_{1}^{(2,1, \mathrm{wb})}$ describes the lowest-order interaction between the surface waves and topography, indicated by the superscript $\mathrm{wb}$. This convolution-type term redistributes the complex amplitudes over the alongshore wavenumber components, thus correcting the wave ray geometry relative to Snel's law $\dagger$ to account for the lateral variability of the topography; lateral diffraction is accounted for implicitly through the summation of the angular wave components (see also Stamnes 1986; Dalrymple \& Kirby 1988; Dalrymple et al. 1989).

For $m=2$ we find forcing terms quadratic in the free wave components that generally result in components that are coupled or bound to the primary waves; in fact they represent the spectral generalization of the second-order harmonic in a periodic wave train first described by Stokes (1847). We will refer to these components with $m=2$ as bound waves. The non-zero forcing term is $S_{12}^{(2,2)}=\mathrm{i} \mathscr{D}_{1,2} \varphi_{1}^{(1,1)} \varphi_{2}^{(1,1)}$, where $\mathscr{D}_{1,2}$ is a quadratic wave-wave interaction coefficient given in Appendix A. The corresponding velocity potential is given by

$$
\phi_{12}^{(2,2)}=\varphi_{12}^{(2,2)} \frac{\mathrm{Ch} Q_{12}}{\operatorname{Ch} q_{12}}, \quad \varphi_{12}^{(2,2)}=\mathrm{i} \frac{\mathscr{D}_{1,2}}{g k_{12} T_{12} \widehat{\Delta}_{12}} \varphi_{1}^{(1,1)} \varphi_{2}^{(1,1)} .
$$

$\dagger$ This is sometimes spelled as 'Snell's law'. The quantitative law of refraction was discovered by the Dutch scientist Willebrord Snel van Royen. His name was latinized into Snellius and later incorrectly de-latinized into Snell. Therefore, the spelling 'Snel's law' is historically appropriate. See also Dingemans (1997, p. 67). 
Expressions (3.8) are in agreement with Hasselmann (1962, §4).

\subsection{Third order, $O\left(\epsilon^{3}\right)$}

At this order, for $m=1$, we incorporate modulations due to the non-stationarity, large-scale bottom slope, cubic wave-wave and wave-bottom interactions, and the interaction with the mean flow. After some algebraic manipulation, the solvability condition (2.23) at this order yields

$$
\begin{aligned}
\mathscr{L}^{(2)}\left\{\varphi_{1}^{(1,1)}\right\}=\xi_{1}^{(3,1, \mathrm{wb})}+\xi_{1}^{(3,1, \mathrm{wbb})}+ & \xi_{1}^{(3,1, \mathrm{wc})}+\xi_{1}^{(3,1, \mathrm{www})} \\
& -\frac{1}{\omega_{1}} \sum_{\boldsymbol{v}_{2}, v_{3}, v_{4}} \mathscr{D}_{2,34} \varphi_{2}^{(1,1)} \varphi_{34}^{(2,2)} \delta_{234 ; 1}^{\lambda, \omega} E_{234 ; 1}
\end{aligned}
$$

where the forcing terms $\xi_{1}^{(3,1, j)}$ are specified below,

$$
\begin{gathered}
\mathscr{L}^{(2)}\{\}=\left[\partial_{t_{2}}+V_{1} \partial_{x_{2}}+\frac{1}{2} \partial_{x_{2}} V_{1}-\frac{\mathrm{i}}{2} M_{1} \partial_{x_{1}}^{2}\right], \\
M_{1}=\left(\frac{\chi_{1}}{k_{1}}\right)^{2} \frac{\partial^{2} \omega_{1}}{\partial k_{1}^{2}}+\left(1-\left(\frac{\chi_{1}}{k_{1}}\right)^{2}\right) \frac{C_{g, 1}}{k_{1}},
\end{gathered}
$$

and we make use of the notional contractions

$$
E_{i j k ; l}=E_{i j k} E_{l}^{*}, \quad \delta_{i j k ; l}^{\lambda, \omega}=\delta\left(\lambda_{i}+\lambda_{j}+\lambda_{k}-\lambda_{l}\right) \delta\left(\omega_{i}+\omega_{j}+\omega_{k}-\omega_{l}\right)
$$

with $\delta$ representing a discrete Dirac delta or unit impulse function (see e.g. Oppenheim \& Schafer 1989).

Equation (3.9) is the third-order transport equation for the free-wave components. The left-hand-side operator in (3.9) includes the linear effects of shoaling, refraction and diffraction for waves propagating over an alongshore-uniform topography $(\bar{h})$. The forcing terms on the right-hand side of (3.9) take into account the third-order effects of the weakly two-dimensional topography $(\widetilde{h})$ and nonlinearity on the wave evolution. In particular, $\xi_{1}^{(3,1, \mathrm{wb})}$ and $\xi_{1}^{(3,1, \mathrm{wbb})}$ account for the interaction with the lateral depth variations; both terms are linear in the surface elevation while linear and quadratic in the bottom perturbation respectively. The terms $\xi_{1}^{(3,1, \mathrm{wc})}$ and $\xi_{1}^{(3,1, \mathrm{www})}$ describe the interaction with the mean flow and cubic nonlinear wave interactions respectively. Note that there is no wave-wave-bottom (wwb) forcing term in (3.9) because in the present approximation the bottom wavenumbers are small relative to the wavenumber mismatch of quadratically forced bound waves and thus result in non-secular contributions.

The convolution term on the right-hand side of (3.9) represents the quadratic interaction between bound and free wave components, which is given in explicit form for later use. Since (3.9) represents an intermediate result, explicit expressions for the remaining forcing terms are omitted for brevity. If needed they can be recovered from the final expressions in physical coordinates and variables which are included in Appendix B.

We combine (3.6) and (3.9) while absorbing the small parameters so that in physical coordinates and variables we have

$$
\mathscr{L}\left\{\varphi_{1}^{(\mathrm{f})}\right\}=\xi_{1}^{(\mathrm{f}, \mathrm{wb})}+\xi_{1}^{(\mathrm{f}, \mathrm{wbb})}+\xi_{1}^{(\mathrm{f}, \mathrm{wc})}+\xi_{1}^{(\mathrm{f}, \mathrm{www})}-\frac{1}{\omega_{1}} \sum_{v_{2}, v_{3}, v_{4}} \mathscr{D}_{2,34} \varphi_{2}^{(\mathrm{f})} \varphi_{34}^{(\mathrm{b})} \delta_{234 ; 1}^{\lambda, \omega} E_{234 ; 1}
$$


where $\varphi_{1}^{(\mathrm{f})}=\epsilon \varphi_{1}^{(1,1)}$ and $\varphi_{34}^{(\mathrm{b})}=\epsilon^{2} \varphi_{34}^{(2,2)}$ and the superscripts $\mathrm{f}$ and $\mathrm{b}$ refer to free and bound waves respectively; the operator $\mathscr{L}\{\}=\epsilon \mathscr{L}^{(1)}\{\}+\epsilon^{2} \mathscr{L}^{(2)}\{\}$. The forcing terms $\xi_{1}^{(\mathrm{f}, j)}$ are equivalent to the $\xi_{1}^{(j)}$ given in Appendix B with $\varphi_{1}$ consistently replaced by $\varphi_{1}^{(\mathrm{f})}$.

\section{Extension to shallow water}

The main result of $\S 3$, the transport equation (3.13), is valid in deep-intermediate water depth but includes a quadratic wave-wave interaction term that involves the second-order bound waves. Closure by means of substitution of expressions (3.8) (in physical variables) for the bound wave amplitudes renders (3.13) incompatible with the limit $O\left(\mu^{2}\right) \rightarrow O(\epsilon)$ where the quadratic interactions approach resonance. To include this limit with $O\left(\epsilon^{2}\right)$ accuracy we extend our expansion of the bound components to higher order, taking into account terms that are of $O\left(\epsilon^{3} / \mu^{2}\right), O\left(\epsilon^{4} / \mu^{4}\right)$, i.e. all terms that are $O\left(\epsilon^{2}\right)$ or lower order as $O\left(\mu^{2}\right) \rightarrow O(\epsilon)$. Although this selective inclusion of higher-order terms may appear to conflict with the expansion for deep-intermediate depth, the ordered expansion is merely a device to make the relative magnitude of terms explicit and is used here to identify terms that can formally be neglected in deepintermediate depth (where they are smaller than the general order of approximation) but are essential in shallow regions where the Stokes number is $O(1)$.

Here we consider the shallow-water limit where $L_{v}=\bar{h}_{0}, \epsilon=a / h$ and use the Boussinesq approximation $O\left(\mu^{2}\right) \sim O(\epsilon)$. Secular contributions from quadratic interactions to the wave field evolution are removed by introducing the composite bound wave amplitude

$$
\varphi_{1}^{(2,2)}=\sum_{\boldsymbol{v}_{2}, \boldsymbol{v}_{3}} \varphi_{23}^{(2,2)} \delta_{23 ; 1}^{\lambda, \omega} E_{23 ; 1}
$$

which can be considered the 'bound' wave contribution to spectral component $\boldsymbol{v}_{1}$. In shallow water, where the forcing is secular, $\varphi_{1}^{(2,2)}$ is a slowly varying quantity that can be described using the same WKB approximation that was used to derive the evolution equations for $\varphi^{(1,1)}$ (namely (3.6), (3.9)). Collecting terms of $O\left(\epsilon^{3} / \mu^{3}\right)$ and $O\left(\epsilon^{4} / \mu^{4}\right)$ (that become $O\left(\epsilon^{3 / 2}\right)$ and $O\left(\epsilon^{2}\right)$ in shallow water respectively) yields forcing terms $R_{1}^{(3,2)}, S_{1}^{(3,2)}, F_{1}^{(3,2)}$ and $R_{1}^{(4,2)}, S_{1}^{(4,2)}, F_{1}^{(4,2)}$ that - apart from the wavewave and wave-current interactions in $S_{1}^{(4,2)}$ - are identical to $R_{1}^{(2,1)}, S_{1}^{(2,1)}, F_{1}^{(2,1)}$ and $R_{1}^{(3,1)}, S_{1}^{(3,1)}, F_{1}^{(3,1)}$ respectively with $\varphi_{1}^{(1,1)}$ and $\varphi_{1}^{(2,1)}$ consistently replaced by $\varphi_{1}^{(2,2)}$ and $\varphi_{1}^{(3,2)}$. For brevity we omit the details (which are similar to those in the analysis for deep-intermediate water) and give the resulting evolution equations for $\varphi_{1}^{(2,2)}$. At $O\left(\epsilon^{3} / \mu^{3}\right)$ we obtain

$$
\mathscr{L}^{(1)}\left\{\varphi_{1}^{(2,2)}\right\}=\xi_{1}^{(3,2, \mathrm{wb})}
$$

with $\xi_{1}^{(3,2, \mathrm{wb})}$ identical to $\xi_{1}^{(2,1, \mathrm{wb})}$ with $\varphi_{1}^{(1,1)}$ replaced by $\varphi_{1}^{(2,2)}$. At $O\left(\epsilon^{4} / \mu^{4}\right)$ we find

$$
\mathscr{L}^{(2)}\left\{\varphi_{1}^{(2,2)}\right\}=\xi_{1}^{(4,2, \mathrm{wb})}+\xi_{1}^{(4,2, \mathrm{wbb})}-\frac{1}{2 \omega_{1}} \sum_{\boldsymbol{v}_{2}, v_{3}} \mathscr{D}_{2,3} \varphi_{2}^{(2,2)} \varphi_{3}^{(2,2)} \delta_{23 ; 1}^{\lambda, \omega} E_{23 ; 1}
$$

The $\xi_{1}^{(4,2, j)}$ on the right-hand side of (4.3) are identical to $\xi_{1}^{(3,1, j)}$ on the right-hand side of (3.9) again with $\varphi_{1}^{(1,1)}$ consistently replaced by $\varphi_{1}^{(2,2)}$. Equations (4.2), (4.3) are higher-order expressions describing the evolution of the $\varphi_{1}^{(2,2)}$ on the slow scales; these expressions are needed solely in the near-resonant limit (shallow water), which justifies the premise of slow variation of the component amplitudes $\varphi_{1}^{(2,2)}$. The similarity with 
(3.6), (3.9) is expected since they are obtained through a similar analysis (WKB); differences are due to the fact that we pursue only $O\left(\epsilon^{2}\right)$ accuracy in shallow water (in contrast to deep-intermediate water where we retain $O\left(\epsilon^{3}\right)$ accuracy), and the quadratic interactions in (4.3) involve a product of bound waves (as opposed to the free wave-bound wave interaction in the corresponding term in (3.9)).

It should be noted that the quadratic response may also contain fast modulations due to large resonance mismatches, even in shallow water for non-collinear interactions. The governing evolution equation, valid from deep-intermediate to shallow water, is obtained by combining (4.2) and (4.3), changing to physical coordinates while accounting for variations on the fast space scale related to the resonance mismatch, and utilizing expressions (3.8) for the bound wave amplitudes. In physical coordinates and variables we have

$$
\mathscr{L}\left\{\varphi_{1}^{(\mathrm{b})}\right\}=\xi_{1}^{(\mathrm{b}, \mathrm{wb})}+\xi_{1}^{(\mathrm{b}, \mathrm{wbb})}-V_{1} \sum_{v_{2}, v_{3}} \frac{\mathscr{D}_{2,3}}{g J_{2,3}}\left(\varphi_{2}^{(\mathrm{f})} \varphi_{3}^{(\mathrm{f})}+\frac{g J_{2,3}}{2 \omega_{1} V_{1}} \varphi_{2}^{(\mathrm{b})} \varphi_{3}^{(\mathrm{b})}\right) \delta_{23,1}^{\lambda, \omega} E_{23 ; 1}
$$

where $\varphi_{1}^{(\mathrm{b})}=\epsilon^{2} / \mu^{2} \varphi_{1}^{(2,2)}, J_{2,3}=k_{23} T_{23} \Delta_{23} / \Lambda_{2,3}$ and $\Lambda_{2,3}=\varkappa_{2}+\varkappa_{3}-\varkappa_{2+3}$. The forcing terms $\xi_{1}^{(\mathrm{b}, j)}$ (involving wave-bottom interaction) are equivalent to the $\xi_{1}^{(j)}$ given in Appendix B with $\varphi_{1}$ consistently replaced by $\varphi_{1}^{(\mathrm{b})}$, indicated by the $\mathrm{b}$ in the superscript.

\section{A generalized third-order evolution model}

The evolution equations (3.13) and (4.4) represent a coupled set of equations for the free and bound wave components respectively. A transport equation for the composite amplitude $\varphi_{1}=\varphi_{1}^{(\mathrm{f})}+\varphi_{1}^{(\mathrm{b})}$ is obtained by summing (3.13) and (4.4):

$$
\begin{aligned}
\mathscr{L}\left\{\varphi_{1}\right\}= & \xi_{1}^{(\mathrm{wb})}+\xi_{1}^{(\mathrm{wbb})}+\xi_{1}^{(\mathrm{wc})}+\xi_{1}^{(\mathrm{www})} \\
& -V_{1} \sum_{\boldsymbol{v}_{2}, v_{3}} \frac{\mathscr{D}_{2,3}}{g J_{2,3}}\left(\varphi_{2}^{(\mathrm{f})} \varphi_{3}^{(\mathrm{f})}+\frac{g J_{2,3}}{2 \omega_{1} V_{1}} \varphi_{2}^{(\mathrm{b})} \varphi_{3}^{(\mathrm{b})}\right) \delta_{23,1}^{\lambda, \omega} E_{23 ; 1} \\
& -\frac{1}{\omega_{1}} \sum_{\boldsymbol{v}_{2}, v_{3}, v_{4}} \mathscr{D}_{2,34} \varphi_{2}^{(\mathrm{f})} \varphi_{34}^{(\mathrm{b})} \delta_{234 ; 1}^{\lambda, \omega} E_{234 ; 1} .
\end{aligned}
$$

The forcing terms $\xi_{1}^{(j)}$ are given in explicit form in Appendix B. Note that

$$
\lim _{\mu^{2} \rightarrow \epsilon} \frac{g J_{2,3}}{2 \omega_{2+3} V_{2+3}}=1+O(\epsilon)
$$

so that, upon substituting $\varphi_{1}$ in the products in the first convolution sum on the $R H S$ of (5.1), we can replace $\varphi_{1}^{(\mathrm{f})}$ by $\varphi_{1}$ and substitute the second-order bound wave solution (3.8) for $\varphi_{34}^{(b)}$ in the remaining terms. These operations result in the closed-form equation

$$
\mathscr{L}\left\{\varphi_{1}\right\}=\xi_{1}^{(\mathrm{wb})}+\xi_{1}^{(\mathrm{wbb})}+\xi_{1}^{(\mathrm{wc})}+\xi_{1}^{(\mathrm{M}, \mathrm{www})}+\xi_{1}^{(\mathrm{ww})}
$$

which retains $O\left(\epsilon^{2}\right)$ accuracy in the limit $O\left(\mu^{2}\right) \rightarrow O(\epsilon)$. Here $\xi^{(\mathrm{ww})}$ and $\xi^{(\mathrm{M}, \mathrm{www})}$ account for quadratic and cubic interactions in $\varphi_{1}$; the latter is modified with respect to the finite depth result, indicated by the $\mathrm{M}$ in the superscript (explicit expressions are given in Appendix B); it ensures proper cubic nonlinearity in finite depth while retaining $O\left(\epsilon^{2}\right)$ accuracy in shallow water. Equation (5.3), the main result of the present analysis, is a one-dimensional (in space) evolution equation for weakly nonlinear waves propagating from deep to shallow water over weakly two-dimensional topography. Although (5.3) does not consider bound and free wave components explicitly, it fully accounts for near-resonant cubic interactions in deep-intermediate 
depth and near-resonant quadratic interactions in shallow water. It is a closed set of equations, apart from the term involving the mean-flow quantities, for which expressions are given in Appendix C. Since the mean flow is modelled without $z$ dependence the model is suitable for water depths where waves 'feel the bottom'. In deeper water $\left(\epsilon k_{0} \bar{h}_{0} \geqslant O(1)\right)$ more general expressions for the mean flow are needed, which is considered in Peregrine (1983).

Equation (5.3) describes the evolution of the velocity potential amplitude. The corresponding spectral amplitudes for the surface elevation function can be readily obtained from

$$
\zeta_{1}=\mathrm{i} \frac{\omega_{1}}{g} \varphi_{1}-\frac{1}{g} \sum_{\boldsymbol{v}_{2}, v_{3}} \mathscr{R}_{2,3} \varphi_{2} \varphi_{3} \delta_{2,3 ; 1}^{\lambda, \omega} E_{2,3 ; 1}
$$

where the nonlinear term is a second-order correction required to accurately include second-order bound waves in the free-surface elevation. This term is $O\left(\epsilon^{2}\right)$ and thus a genuine second-order term irrespective of the resonance mismatch. In contrast, the contribution to $\varphi_{1}$ due to quadratic interactions is $O\left(\epsilon^{2} / \mu^{2}\right)$ and becomes an $O(\epsilon)$ secular forcing term in shallow water with leading-order contributions over distances $O\left(\epsilon^{-1}\right)$. In view of this, the nonlinear term in (5.4) - contributing a local, second-order correction without affecting the wave field evolution - is usually neglected in models for near-resonant quadratic interactions (e.g. Agnon et al. 1993; Kaihatu \& Kirby 1995), which is consistent with the premise of that derivation. Since the present model allows for arbitrary resonance mismatch (and thus includes off-resonant, secondorder bound waves), the inclusion of the nonlinear term in (5.4) is necessary to obtain second-order accuracy in the surface elevation matching Stokes' second-order theory (see also Bredmose et al. 2002).

In the following subsections we describe special cases of the model presented here, for deep-intermediate $(\S 5.1)$ and shallow $(\S 5.2)$ water and compare the results with expressions published previously.

\subsection{Deep-intermediate water}

We consider wave propagation in deep-intermediate water $\left(k_{0} \bar{h}_{0} \sim O(1)\right)$, such that quadratic interactions result in second-order bound waves and nonlinear resonances are accounted for through the cubic forcing term $\xi^{(\mathrm{www})}$ given in Appendix B. It can be shown through some algebraic manipulation that the interaction coefficient $\mathscr{H}_{1,2,3}$ in $\xi^{(\mathrm{www})}$ is equivalent to that of Herterich \& Hasselmann (1980) (a corrected version of the interaction term given in Hasselmann 1962). Furthermore, it can be shown that prediction of the dispersion corrections from the pairwise, third-order interaction of a bichromatic directional wave train in deep water agree with expressions derived by Longuet-Higgins \& Phillips (1962) (when accounting for the misprint in that paper previously pointed out by Hogan, Gruman \& Stiassnie (1988) and Willebrand (1973, 1975)).

If we further restrict the wave field to a single angular component

$$
\eta=\zeta \exp \left[\mathrm{i}\left(\int^{x} x \mathrm{~d} x+\lambda y-\omega t\right)\right]+*,
$$

where $*$ denotes the complex conjugate of the preceding term, then (5.3) reduces to

$$
\begin{aligned}
& {\left[\partial_{t}+V \partial_{x}+\frac{1}{2} \partial_{x} V-\frac{\mathrm{i}}{2}\left[\left(\frac{x}{k}\right)^{2} \frac{\partial^{2} \omega}{\partial k^{2}}+\left(1-\left(\frac{\varkappa}{k}\right)^{2}\right) \frac{C_{g}}{k}\right] \partial_{x}^{2}\right] \zeta} \\
& =-\mathrm{i} \frac{k^{2} \omega}{4 T^{2}}\left(9 T^{-2}-12+13 T^{2}-2 T^{4}\right)|\zeta|^{2} \zeta+\mathrm{i} \zeta\left[\frac{k^{2}}{2 \omega}\left(1-T^{2}\right) \partial_{t}-\boldsymbol{k} \cdot \nabla\right] \bar{\Phi}
\end{aligned}
$$


This expression, coupled to the mean-flow equations in Appendix C, agrees with corresponding expressions given by e.g. Liu \& Dingemans (1989) if likewise lateral modulation of the wave amplitude is omitted and higher-order refraction corrections (included in Liu \& Dingemans 1989) are excluded. For small-angle wave propagation over a horizontal bottom (5.3), along with the expressions for the mean-flow in Appendix C, can readily be written in the form of Davey \& Stewartson (1974).

For one-dimensional wave propagation $\left(\varkappa_{i}=k_{i}\right)$ in deep-intermediate water depth, (5.6) can be shown to agree with expression (2.14) in Djordjevic \& Redekopp (1978) (if the last $\sigma$ in their expression (2.17) is replaced by $\sigma^{2}$ ) and with the corresponding expressions given in Mei $(1983, \S 12)$. For the reduced case of Stokes waves, i.e. onedimensional periodic waves of permanent shape in uniform depth, the model is in agreement with the expressions derived by Stokes (1880) to third order in wave steepness.

\subsection{Shallow water}

When considering the propagation of steady waves over alongshore-uniform bathymetry while omitting interaction with the mean flow and cubic wave-wave interactions (thus implicitly restricting the model to fairly shallow water), the evolution equation (5.3) is equivalent to that in Bredmose et al. (2002). It represents a one-dimensional generalization, with respect to the treatment of the quadratic interactions, of the earlier models by Agnon et al. (1993), Kaihatu \& Kirby (1995), Sheremet (1996), and Eldeberky \& Madsen (1999).

Taking the shallow-water limit of (5.3) using the Boussinesq scaling $O\left(\epsilon / \mu^{2}\right)=O(1)$, yields to lowest order the models of Herbers \& Burton (1997) and (for unidirectional waves) Freilich \& Guza (1984, the 'consistent shoaling model').

\section{Numerical evaluation and comparison to data}

In this section we compare model results with laboratory observations of steady unidirectional waves to verify the generalized validity of the nonlinear interaction terms that account for cubic resonances in deep-intermediate water and quadratic resonances in shallow water. Additionally, a comparison with observations of wave propagation over two-dimensional topography is presented to validate the model's ability to describe accurately the combined effects of refraction, lateral diffraction and harmonic generation.

For a steady wave field (5.3) reduces to

$$
\left(\frac{\mathrm{d}}{\mathrm{d} x}+\frac{1}{2 V_{1}} \frac{\mathrm{d} V_{1}}{\mathrm{~d} x}\right) \varphi_{1}=\Xi_{1}^{(\mathrm{wb})}+\Xi_{1}^{(\mathrm{wbb})}+\Xi_{1}^{(\mathrm{ww})}+\Xi_{1}^{(\mathrm{www})}+\Xi_{1}^{(\mathrm{wc})}
$$

where $\Xi_{1}^{(\mathrm{ww})}=\xi_{1}^{(\mathrm{ww})} / V_{1}, \Xi_{1}^{(\mathrm{www})}=\xi_{1}^{(\mathrm{M}, \mathrm{www})} / V_{1}$ and the remaining forcing terms are given in Appendix B. The corresponding surface elevation amplitudes, $\zeta_{1}$, are found through (5.4).

\subsection{One-dimensional wave propagation}

For the cases of one-dimensional wave propagation we numerically evaluate (6.1) without the terms involving the lateral depth variability, i.e. for $\lambda=0$. The forcing term $\Xi_{1}^{(\mathrm{wc})}=\Xi_{p_{1}, 0}^{(\mathrm{wc})}$ is given as

$$
\Xi_{p_{1}, 0}^{(\mathrm{wc})}=\mathrm{i} \frac{\varkappa_{p_{1}, 0}}{V_{p, 0}} \varphi_{p_{1}, 0} \sum_{p_{2}} \frac{\omega_{p_{2}} \varkappa_{p_{2}, 0}}{g h}\left|\varphi_{p_{2}, 0}\right|^{2} .
$$


The $\Xi_{p_{1}, 0}^{(\mathrm{wc})}$ accounts for the effects of the attendant return current - to ensure zero net mass flux - on the wave propagation. The predicted wave-induced water level changes (i.e. set-down) were found to be everywhere smaller than $0.1 \%$ of the undisturbed water depth, and are neglected here.

The model is initialized at the up-wave boundary with the observed primary spectral component(s) obtained from a Fourier-transformed time series at that location with added (theoretical) second-order harmonics. This boundary condition was chosen, instead of simply utilizing the full observed spectrum, to minimize spurious modulations in the computations induced by spectral leakage effects as a result of small imperfections in e.g. wave generation or recording devices (see also Shemer et al. 2001).

The ordinary differential equations (6.1) are integrated using a standard fourthorder, fixed-step-size, Runge-Kutta scheme.

\subsubsection{Evolution of wave groups in intermediate water depth}

The third-order model (6.1) is compared to observations of periodic wave groups propagating in relatively deep water reported by Shemer et al. (2001), to verify the model's representation of the deep-water nonlinear physics (i.e. near-resonant cubic interactions). The experiments were conducted in a wave flume, $18 \mathrm{~m}$ long and $1.2 \mathrm{~m}$ wide, with a uniform water depth of $0.60 \mathrm{~m}$. The positive $x$-axis is in the direction of propagation with the origin at the wave generator. For more detailed information on the experimental set-up and the complete set of experiments conducted we refer to Shemer et al. (1998, 2001).

The wave field consists of a modulated carrier wave with period $T_{0}=0.9 \mathrm{~s}$. The wave board is driven by a signal of the form

$$
s(t)=s_{0}\left|\cos \left(\Omega_{0} t\right)\right| \cos \left(\omega_{0} t\right), \quad \Omega_{0}=\frac{\omega_{0}}{20},
$$

where $\omega_{0}=2 \pi / T_{0}$. The spectrum of this signal is characterized by a maximum at $\omega_{0}$ and sidebands at integer multiples of $2 \Omega_{0}$ with the two nearest to $\omega_{0}$ being most significant. For the case considered here $k_{0} a_{0} \approx 0.21$, where $k_{0}$ is related to $\omega_{0}$ through the linear dispersion relation and $a_{0}$ is taken (after Shemer et al. 1998) as the maximum amplitude of the carrier wave in a group close to the wave generator.

The model is initialized with the spectral components at $\omega_{0}$ and $\omega_{0} \pm 2 \Omega_{0}$ of a time series of $18 \mathrm{~s}$ (i.e. 20 wave periods) duration observed at $x=0.245 \mathrm{~m}$ (re-sampled with $N=1024$ points and $\Delta t=0.0176 \mathrm{~s}$ ). Second-order components are computed using Stokes' second-order theory (equation (3.8)) and included in the up-wave boundary condition. We compute the evolution of an equidistant array of 65 frequencies with $\Delta \omega=2 \pi /(N \Delta t)$. The integration is performed using a step size, $\Delta x$, of $0.1 \mathrm{~m}$.

In figure 1 we compare the observed (circles) and predicted (solid line) time series at four positions: $x=\{0.245,1.845,6.98,8.425\} \mathrm{m}$. The initially near-symmetrical wave groups develop strong left-right asymmetry of the envelope with steep fronts and gently sloping rears as also observed in the experiments reported by e.g. Feir (1967). The asymmetry can be explained heuristically by the combined effects of amplitude and frequency dispersion (Lighthill 1978, p. 462). Initially the larger waves in the group propagate fastest under the effects of amplitude dispersion, reducing wavelengths in the front of the group and increasing those in the rear; the associated group speed variation further enhances the localization of energy in the front of the groups resulting in increasingly forward leaning of the wave groups as observed in the experimental data. 

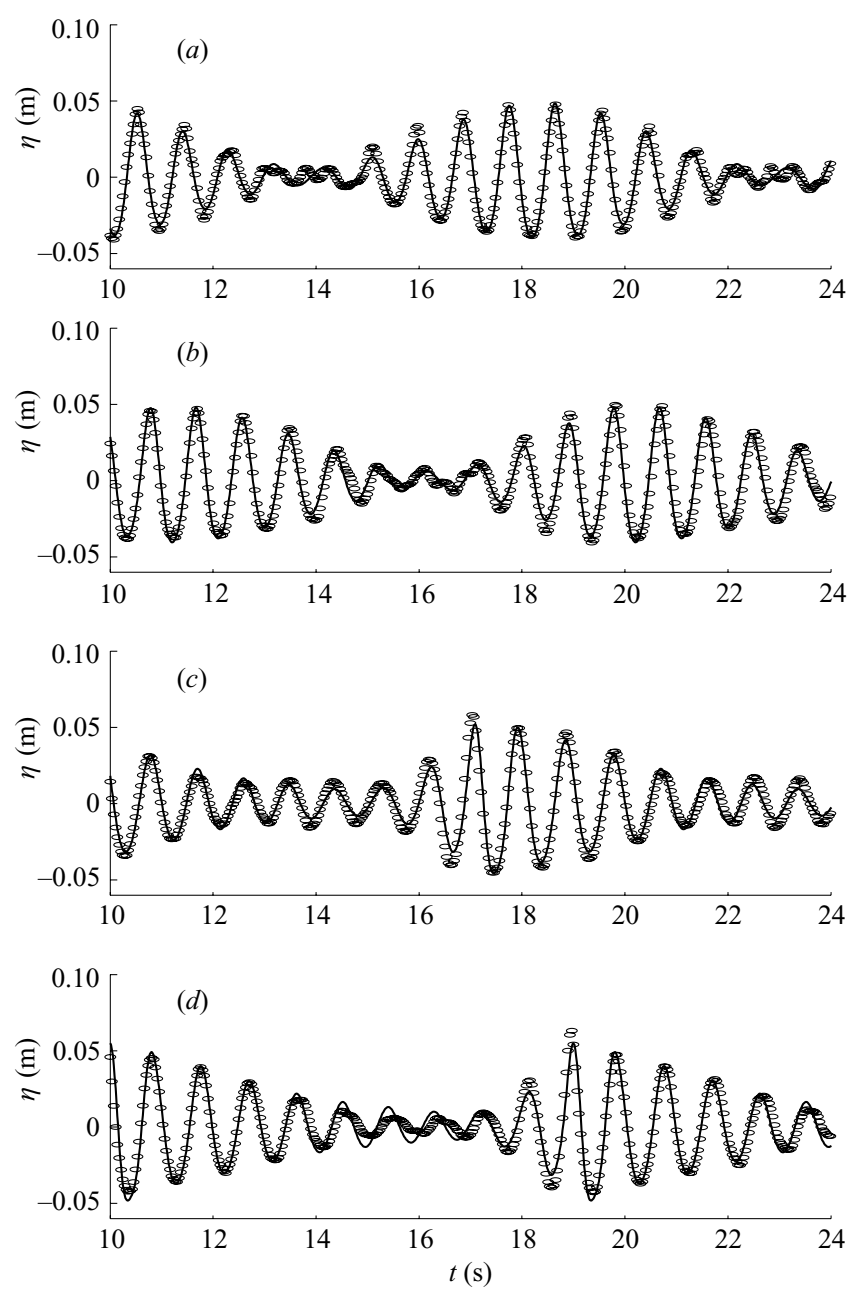

FIGURE 1. Time series comparison of observed and predicted nonlinear wave group evolution in uniform depth. The carrier frequency $\omega_{0}=2 \pi /(0.9 \mathrm{~s})$, water depth $=0.6 \mathrm{~m}, \epsilon \approx 0.21$. Circles denote observed surface elevations from Shemer et al. $(1998,2001)$; solid line denotes model result. Panels $(a)-(d)$ correspond to positions $\{0.245,1.845,6.98,8.425\} \mathrm{m}$.

The model predictions accurately reproduce both the increasing asymmetry of the envelope and the evolution of the individual waves. Apart from some discrepancies in the less energetic region between groups, the agreement is excellent; this demonstrates the model's ability to accurately predict wave group evolution resulting from nearresonant cubic wave-wave interactions in deep-intermediate water depth.

Model simulations (not shown here) extended to larger distances from the wavemaker than for which observations were available in the experimental data, indicate that the groups eventually split into a group consisting of the higher waves and one with the lower waves. The higher-wave group leads and eventually coalesces with the preceding low-wave group followed by recurrence-type behaviour qualitatively similar to that observed and discussed in Lo \& Mei (1985). Intermittent wave breaking due to increased steepness ( $k_{0} a \approx 0.34$ was observed in the numerical simulations) will probably result in only partial recurrence. 


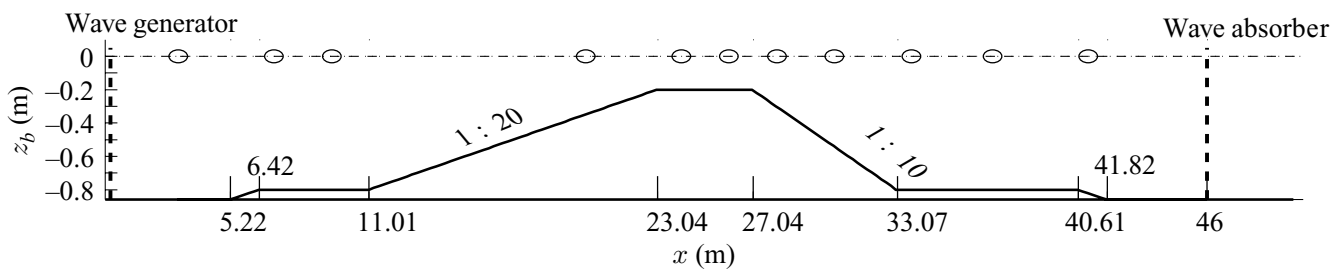

FIGURE 2. Schematic representation of the trapezoidal bottom profile of Luth et al. (1994); circles at $z_{b}=0$ denote wave gauge positions.

\subsubsection{Wave propagation over a submerged obstacle}

To test the model's ability to predict harmonic generation due to near-resonant quadratic interactions over variable depth in relatively shallow water, we consider the propagation of waves over a trapezoidal shoal in a laboratory flume (Luth, Klopman \& Kitou 1994; Beji \& Battjes 1993), a schematic representation of which is shown in figure 2. The up- and downslope of the shoal are 1:20 and 1:10 respectively. Away from the shoal the uniform water depth is $0.80 \mathrm{~m}$, and the minimum depth over the shoal is $0.20 \mathrm{~m}$. The wave generator is positioned at $x=0$ and at $x=46 \mathrm{~m}$ a wave absorber was installed to ensure uni-directional wave propagation in the area of interest; circular markers in the figure denote wave gauge positions. The bathymetrical dimensions are those reported by Luth et al. (1994), which is a scaled version (by a factor of 2) of the set-up used by Beji \& Battjes (1993). The wave field characteristics were scaled accordingly to reproduce cases reported by Beji \& Battjes (1993).

The incident wave train is monochromatic with period $T_{0}=2.86 \mathrm{~s}$ and amplitude $0.02 \mathrm{~m}$. The model is initialized with the spectral component at $\omega_{0}$ of a (re-sampled) time series of $25.71 \mathrm{~s}$ duration (512 points at $0.0502 \mathrm{~s}$ intervals) observed at $x=3.04 \mathrm{~m}$. The first harmonic is computed using Stokes' second-order theory (equation (3.8)) and included in the up-wave boundary condition.

The numerical integration is performed for 60 equidistant frequency components with $\Delta \omega=2 \pi /(N \Delta t)$ with a spatial step size of $0.1 \mathrm{~m}$.

Figure 3 shows a comparison of observed and predicted surface elevation time series for positions $x=\{20.04,24.04,28.04,30.44,33.64,41.04\} \mathrm{m}$. The model succesfully captures the initial steepening and forward leaning of the waves on the up-slope as well as the enhancement and release of higher harmonics over the shoal and the down-slope; the agreement between observed and predicted time series is excellent, even at the farthest location $x=41.04 \mathrm{~m}$ (figure 3). Similar agreement with somewhat larger discrepancies in the details of the wave profile was reported by Beji \& Battjes (1994), who compared simulations of a Boussinesq model with improved dispersion characteristics (Madsen \& Sørensen 1992) to the earlier dataset of Beji \& Battjes (1993) in a more restricted domain. These authors also illustrated that improved dispersion is crucial to predict the wave evolution accurately by including a comparison to simulations with Boussinesq equations with lowest-order dispersion (Peregrine 1967), which compared considerably less favourably with the observations than the dispersion-enhanced formulation by Madsen \& Sørensen (1992). Dingemans $(1997, \S 5.9)$ showed that even the predictions with the enhanced Boussinesq formulation deteriorate for positions far behind the shoal $(x=33.64 \mathrm{~m}$ and $x=41.04 \mathrm{~m}$, no measurements were available at these positions in the set-up used by Beji \& Battjes), suggesting that taking full account of dispersion (as in the present work) or a high-order approximation (e.g. Agnon, Madsen \& Schäffer 1999; 

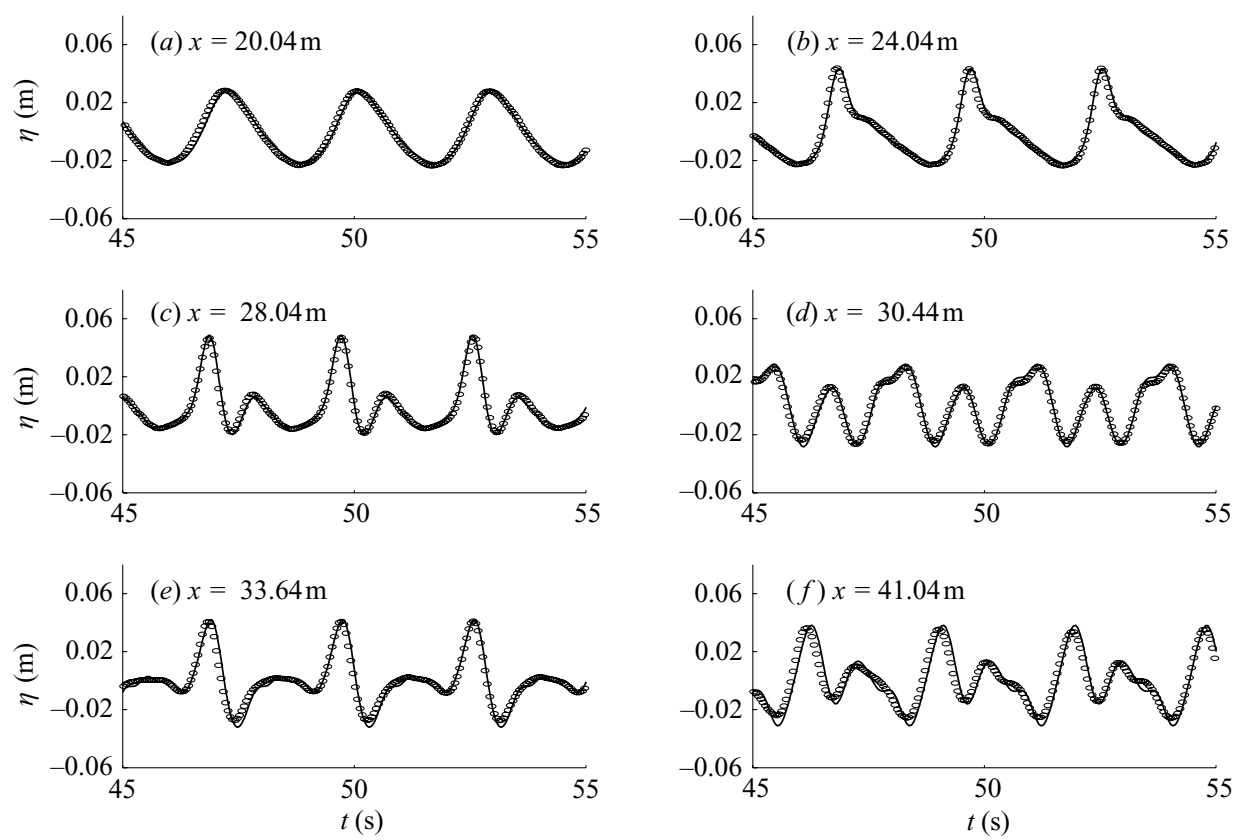

FIGURE 3. Comparison observed and predicted surface elevation time series for wave propagation over a shoal. Incident waves have period $2.86 \mathrm{~s}$ and amplitude $0.02 \mathrm{~m}$. Circles denote laboratory observations by Luth et al. (1994), solid line represents model predictions.

Madsen, Bingham \& Schäffer 2003) is essential to resolve wave phases over larger distances.

Although the depth variations over the shoal are fairly rapid, the observations at locations between the generator and the trapezoid (not shown here) did not indicate the presence of significant wave reflections; moreover, the level of agreement between observations and predictions supports the assumptions of unidirectionality and slowly varying depth as implicit in the model.

\subsection{Nonlinear wave propagation over two-dimensional bathymetry}

To validate the model representation of weakly two-dimensional topography, we compare model predictions to observations reported by Whalin (1971) who performed experiments on an alongshore convex-shaped beach shown in figure 4 . The wave generator was positioned in the deeper part of the wave tank at $x=1.615 \mathrm{~m}$. Monochromatic waves were generated; significant enhancement of harmonics was observed in the focal region where the combined effects of refraction, diffraction and nonlinear wave-wave interactions are important.

For this case the waves are in relatively shallow water so that the nonlinear evolution is predominantly due to near-resonant quadratic interactions; cubic interactions are assumed of lesser importance here. The observations are thus compared to simulations performed with a numerical implementation of (6.1), omitting the terms accounting for cubic wave-wave interactions, $\Xi_{1}^{(\mathrm{www})}$, and the interaction with the mean flow, $\Xi_{1}^{(\mathrm{wc})}$.

We consider two cases, both with normally incident waves of $2 \mathrm{~s}$ period $\left(\omega_{0}=\right.$ $\pi \mathrm{rad} \mathrm{s}^{-1}, \lambda_{0}=0$ ) and initial amplitudes of $0.75 \mathrm{~cm}$ and $1.06 \mathrm{~cm}$ respectively (a snapshot 

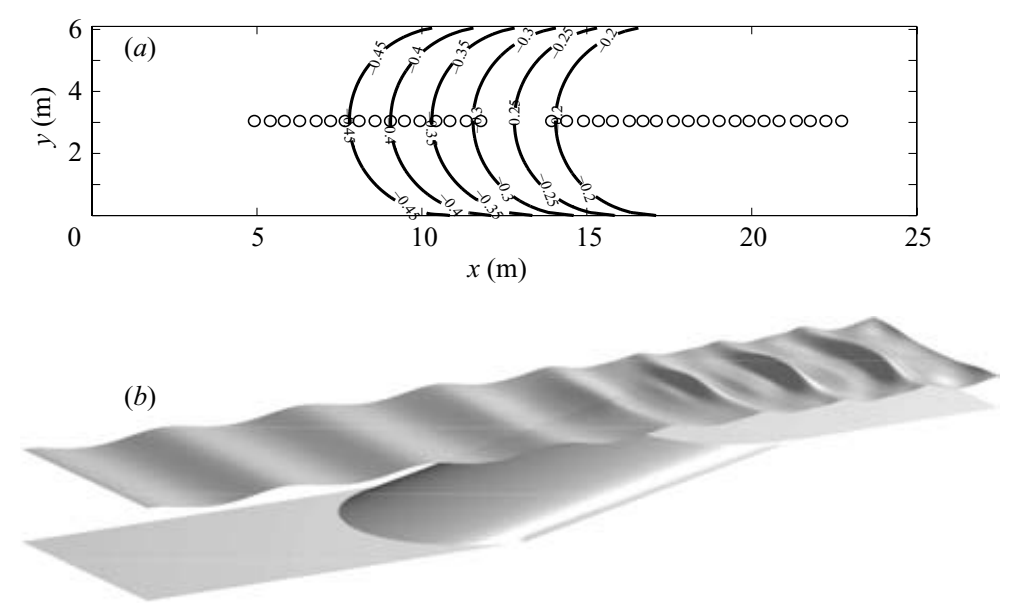

Figure 4. (a) Depth contours experimental set-up of Whalin (1971) (units m), circles denote wave gauge positions along the centreline. $(b)$ Model-predicted surface elevation (equation (6.1)) for incident wave with period $2.0 \mathrm{~s}$ and initial amplitude $1.06 \mathrm{~cm}$.

of the model-predicted surface elevation over the topography for the larger-amplitude case is shown in figure $4 b$ ). The model is initialized with a single spectral component with the appropriate initial amplitude, frequency and alongshore wavenumber at $x=0$ and the corresponding (theoretical) first harmonic. The spatial domain is discretized with $\Delta x$ and $\Delta y$ both equal to $0.1 \mathrm{~m}$. A fourth-order, fixed-step-size, RungeKutta scheme is used to evaluate the evolution of the primary wave $\left(\omega_{0}\right)$ and its three harmonics $\left(2 \omega_{0}, 3 \omega_{0}, 4 \omega_{0}\right)$. Evanescent modes $\left(|\lambda|>k_{\omega}\right)$ are excluded from the computations, including those modes that are evanescent at $x=0$ but become propagating inside the computational domain as a result of the variable depth. The alongshore wavenumber interval $\Delta \lambda=2 \pi / L_{y}$ where $L_{y}$ denotes the lateral extent of the computational domain.

Figure 5 shows the evolution of the spectral amplitudes of the primary component, and the first and second harmonic, for the two cases considered (left and right panels respectively). As the waves propagate from the deep end of the flume over the topography into shallow water, the primary wave component initially increases, predominantly due to shoaling and wave convergence, until quadratic interactions approach resonance resulting in the observed rapid growth of the first and second harmonic at the expense of the primary wave component, followed by partial recurrence. Apart from a predicted spatial oscillation of the primary component for the larger wave case in the down-wave region of the flume (panel $b$ ), which is absent in the observations (a similar discrepancy was invariably found by other authors, e.g. Liu, Yoon \& Kirby 1985; Kaihatu \& Kirby 1995; Tang \& Ouellet 1997), the model predictions are in good agreement with the observations.

\subsection{Numerical efficiency and outlook}

The application of a multi-frequency angular spectrum decomposition results in a dimensional reduction of the governing equation (a set of ODEs describes the evolution of stationary, two-dimensional wave fields over two-dimensional topography) at the expense of convolution-type forcing terms for the wave-wave and wave-bottom interaction, which is typical for spectral Galerkin-type methods. 

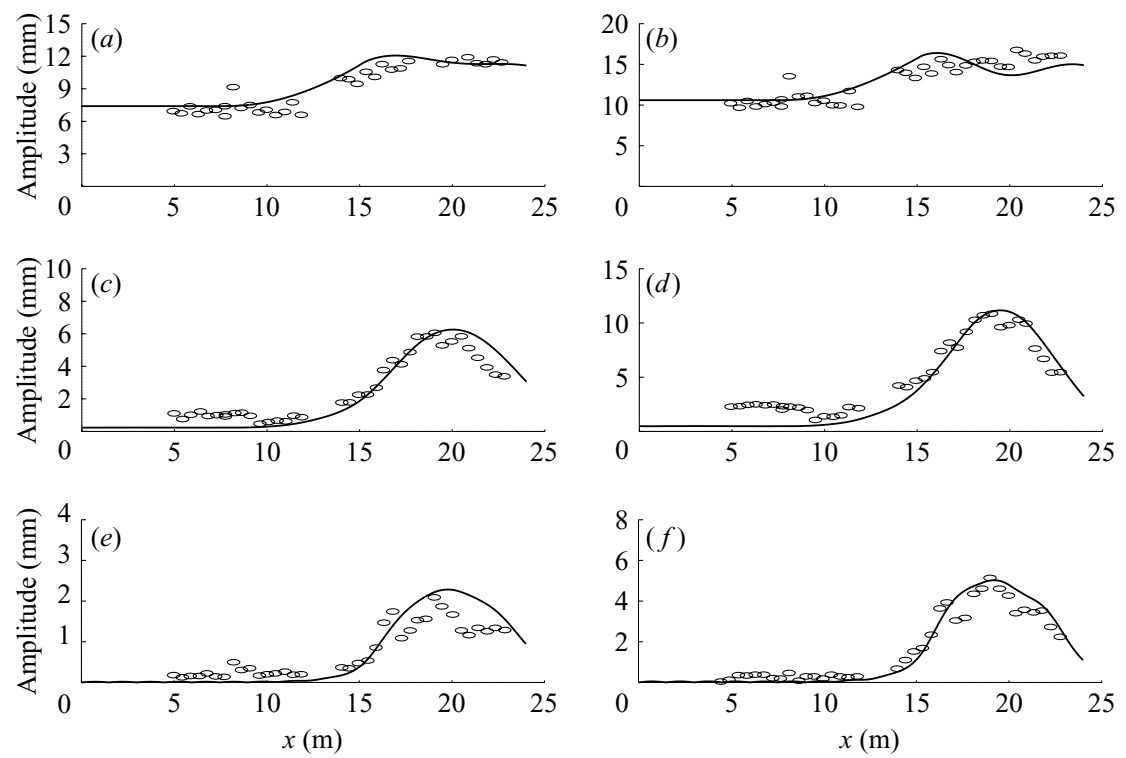

FIGURE 5. Evolution of amplitudes of primary wave component and harmonics for a periodic incident wave with period $2.0 \mathrm{~s}$ propagating over two-dimensional bathymetry. Left panels: initial amplitude $0.75 \mathrm{~cm}$. Right panels: $1.06 \mathrm{~cm}$. $(a, b) \omega_{0},(c, d) 2 \omega_{0},(e, f) 3 \omega_{0}$. Circles denote observed values by Whalin (1971), solid line represents model results. Note differences in amplitude scales.

Direct evaluation of the convolution-type forcing terms is straightforward, but for the cubic interaction terms, for example, the number of operations is $O\left(N^{3}\right)(N$ being the number of spectral components) which becomes prohibitive for larger-scale applications. Such terms can be implemented by utilizing standard pseudospectral methods (see e.g. Orszag 1972) so that the number of operations scales as $O\left(N \log _{2} N\right)$ (see also e.g. Bredmose et al. 2002, 2004; Canuto et al. 1987); this renders the approach suitable for application to wave fields with realistic spectra and spatial scales as encountered in typical (nearshore) oceanographic applications. No such (or other) efficiency improvements were pursued for the numerical evaluations presented here, but we remark that on a standard desktop (P4) computer the computations for the unidirectional cases with cubic nonlinearity take a few minutes, not hours; the twodimensional model for the Whalin experiment (cubic terms omitted and four harmonics computed) requires less than a minute of CPU time.

For waves of moderate amplitude over mildly varying depth with weak lateral variations, the multi-frequency angular spectrum approach presented here is efficient and applicable to scales covering hundreds of wavelengths (e.g. wave propagation over the continental shelf onto beaches); in particular, it has the potential to investigate the effects and relative importance of competing nonlinear processes in random ocean waves over shoals and banks. More general time-domain models such as boundaryintegral methods and field approximations of the Laplace equation (e.g Westhuis 2001) or even recent developments in extended Boussinesq theory (e.g Madsen et al. 2003) are potentially more accurate, in particular over steep topography or in the presence of strong nonlinearity, but are computationally intensive which generally restricts their application to smaller areas. Furthermore, such models do not explicitly describe the wave interaction mechanisms, which, since Stokes (1847), are so deeply 
rooted in our conceptual understanding of nonlinear wave physics and at the basis of widely used operational wave models (e.g. Hasselmann 1962).

For random ocean wave fields, understanding of the evolution of the wave field statistics is often more useful than detailed, deterministic knowledge of individual realizations. Such statistical information can be obtained either through, numerically intensive, Monte Carlo simulations, or by recasting the governing equations to compute ensemble-averaged wave field quantities directly, thus describing the wave field evolution in a stochastic, rather than deterministic, sense (e.g. Herbers \& Burton 1997; Agnon \& Sheremet 1997); the multi-frequency angular spectrum approach is particularly well-suited as a basis for the latter approach.

\section{Conclusions}

Utilizing an angular spectrum decomposition of the wave field, a discrete spectral evolution model was derived for the propagation of nonlinear surface waves over weakly two-dimensional topography. The model accounts for the linear effects of refraction, shoaling, diffraction and non-stationarity as well as quadratic and cubic nonlinear effects. It is a generalization and extension of previous work in the sense that: (i) arbitrary resonance mismatch for quadratic interactions in a multi-frequency, multi-directional wave field propagating over weakly two-dimensional topography is included with exact correspondence to well-known deep-intermediate (Stokes) and shallow-water (Boussinesq) limits; (ii) it accounts for cubic near resonances in deep-intermediate water without suffering the breakdown in shallow water typically associated with Stokes-type expansions.

The equivalence with well-known expressions for nonlinear wave propagation in deep-intermediate water is shown. By taking proper limits, the model is shown to include both narrow-band cubic Schrödinger-type equations and models for quadratic resonant interactions as special cases, while reducing to a classical Boussinesq formulation in shallow water.

Comparisons of numerical simulations to laboratory observations of onedimensional wave propagation demonstrate that the model accurately predicts both cubic nonlinear effects on the evolution of wave groups in relatively deep water, and harmonic generation in relatively long waves propagating over a submerged obstacle. The successful modelling of such disparate nonlinear cases with a single model illustrates the general validity of the present formulation.

Comparisons to laboratory observations of two-dimensional wave propagation over a convex beach confirm the robustness of the weakly two-dimensional approximation of topographical features in describing the combined effects of refraction, diffraction and harmonic generation in a realistic focusing region on a beach.

This research is supported by the Technology Foundation STW, applied science division of NWO and the technology programme of the Ministry of Economic Affairs in the Netherlands, under project number DCB.6025. T. H.C.H. is supported by the Office of Naval Research (Coastal Geosciences Program) and the National Science Foundation (Physical Oceanography Program) in the USA. We are indebted to Lev Shemer, Maarten Dingemans, Gert Klopman, James Kaihatu and Ole Sørensen for generously sharing experimental data and related documentation. The manuscript has benefited from the detailed comments and suggestions for improvement from the anonymous referees and D. H. Peregrine. 


\section{Appendix A. Wave-wave interaction coefficients}

A.1. Quadratic coefficients

$$
\begin{gathered}
\mathscr{D}_{1,2}=-\frac{1}{2}\left[\omega_{1} k_{2}^{2}\left(1-\frac{\omega_{2}^{2}}{g k_{2}} T_{2}\right)+\omega_{2} k_{1}^{2}\left(1-\frac{\omega_{1}^{2}}{g k_{1}} T_{1}\right)-2\left(\omega_{1}+\omega_{2}\right)\left(k_{1} k_{2} T_{1} T_{2}-\boldsymbol{k}_{1} \cdot \boldsymbol{k}_{2}\right)\right], \\
\mathscr{R}_{1,2}=\frac{1}{2 g}\left[\omega_{1} \omega_{2}\left(k_{1} T_{1}+k_{2} T_{2}\right)+g\left(k_{1} k_{2} T_{1} T_{2}-\boldsymbol{k}_{1} \cdot \boldsymbol{k}_{2}\right)\right] .
\end{gathered}
$$

A.2. Cubic coefficients

$$
\mathscr{D}_{1,2,3}=\frac{1}{3}\left[\widehat{\mathscr{D}}_{1,2,3}+\widehat{\mathscr{D}}_{2,1,3}+\widehat{\mathscr{D}}_{3,1,2}\right]
$$

where

$$
\begin{aligned}
\widehat{\mathscr{D}}_{1,2,3}= & \frac{\omega_{1}}{g}\left(\omega_{2}+\omega_{3}\right)\left(\boldsymbol{k}_{2} \cdot \boldsymbol{k}_{3}\left(k_{2} T_{2}+k_{3} T_{3}\right)-k_{3} k_{2}\left(k_{3} T_{2}+k_{2} T_{3}\right)\right) \\
& +\boldsymbol{k}_{1} \cdot\left(\boldsymbol{k}_{2}+\boldsymbol{k}_{3}\right)\left(k_{2} k_{3} T_{2} T_{3}-\frac{1}{2} \boldsymbol{k}_{2} \cdot \boldsymbol{k}_{3}\right)-k_{1}^{2} k_{2} k_{3} T_{2} T_{3}+k_{1}^{2}\left(1-T_{1}^{2}\right) \mathscr{R}_{2,3} .
\end{aligned}
$$

Note that the $\widehat{\mathscr{D}}_{1,2,3}$ are symmetrical in the last two indices so that $\mathscr{D}_{1,2,3}$ is symmetrical in all three indices.

\section{Appendix B. Forcing terms}

The forcing terms on the transport equation (5.1) in physical variables and coordinates are

$$
\begin{aligned}
\xi_{1}^{(\mathrm{wb})}= & \mathrm{i} \frac{g}{2 \omega_{1}}\left(1-T_{1}^{2}\right)\left[k_{1}^{2} \mathscr{G}_{1}\left\{\widetilde{h}, \varphi_{2}\right\}+\mathrm{i} \lambda_{1}\left(q_{1} T_{1}-1\right) \mathscr{G}_{1}\left\{\widetilde{h}_{y}, \varphi_{2}\right\}\right. \\
& \left.+\mathrm{i} \varkappa_{1}\left(\frac{C_{g, 1}}{2 C_{1}}+q_{1} T_{1}-1\right)\left[\mathscr{G}_{1}\left\{\widetilde{h}_{x}, \varphi_{2}\right\}+2 \mathscr{G}_{1}\left\{\widetilde{h}, \varphi_{2, x}\right\}\right]\right], \\
\xi_{1}^{(\mathrm{wbb})}= & \mathrm{i} \frac{g}{2 \omega_{1}}\left(1-T_{1}^{2}\right) \frac{k_{1}^{3}\left(1+3 T_{1}^{2}\right)}{4 T_{1}} \mathscr{G}_{1}\left\{\widetilde{h}^{2}, \varphi_{2}\right\}, \\
\xi_{1}^{(\mathrm{www})}= & \frac{\mathrm{i}}{2 \omega_{1}} \sum_{\boldsymbol{v}_{2}, \boldsymbol{v}_{3}, \boldsymbol{v}_{4}} \mathscr{H}_{2,3,4} \varphi_{2} \varphi_{3} \varphi_{4} \delta_{234 ; 1}^{\lambda, \omega} E_{234 ; 1}, \\
\xi_{1}^{(\mathrm{wc})}= & \mathrm{i} \frac{k_{1}^{2}}{2 \omega_{1}}\left(1-T_{1}^{2}\right) \mathscr{G}_{1}\left\{\overline{\bar{\phi}}_{t}, \varphi_{2}\right\}-\mathscr{G}_{1}\left\{\overline{\bar{\phi}}_{x}, \mathrm{i} \varkappa_{2} \varphi_{2}\right\}-\mathscr{G}_{1}\left\{\overline{\bar{\phi}}_{y}, \mathrm{i} \lambda_{2} \varphi_{2}\right\},
\end{aligned}
$$

where

$$
\mathscr{H}_{1,2,3}=\frac{1}{3}\left[\widehat{\mathscr{H}}_{1,2,3}+\widehat{\mathscr{H}}_{2,1,3}+\widehat{\mathscr{H}}_{3,1,2}\right], \quad \widehat{\mathscr{H}}_{1,2,3}=\widehat{\mathscr{D}}_{1,2,3}-2 \frac{\mathscr{D}_{2,3}}{g k_{23} T_{23} \Delta_{23}} \mathscr{D}_{1,23} .
$$

The quadratic and modified cubic nonlinear term in (5.3) are

$$
\begin{aligned}
\xi_{1}^{(\mathrm{ww})} & =-V_{1} \sum_{\boldsymbol{v}_{2}, \boldsymbol{v}_{3}} \frac{\mathscr{D}_{2,3}}{g J_{2,3}} \varphi_{2} \varphi_{3} \delta_{23 ; 1}^{\lambda, \omega} E_{23 ; 1}, \\
\xi_{1}^{(\mathrm{M}, \mathrm{www})} & =\frac{\mathrm{i}}{2 \omega_{1}} \sum_{\boldsymbol{v}_{2}, \boldsymbol{v}_{3}, \boldsymbol{v}_{4}} \mathscr{W}_{2,3,4} \varphi_{2} \varphi_{3} \varphi_{4} \delta_{234 ; 1}^{\lambda, \omega} E_{234 ; 1},
\end{aligned}
$$


where in (B 7)

$$
\mathscr{W}_{1,2,3}=\frac{1}{3}\left[\widehat{\mathscr{W}}_{1,2,3}+\widehat{\mathscr{W}}_{2,1,3}+\widehat{\mathscr{W}}_{3,1,2}\right]
$$

and

$$
\widehat{\mathscr{W}}_{1,2,3}=\widehat{\mathscr{D}}_{1,2,3}-2 \frac{\mathscr{D}_{2,3}}{g k_{23} T_{23} \Delta_{23}}\left(\mathscr{D}_{1,23}-2 \omega_{1+2+3} V_{1+2+3} \frac{\mathscr{D}_{1,2+3}}{g J_{1,2+3}}\right) .
$$

The forcing terms on the transport equation for a stationary wave field, (6.1), are

$$
\begin{aligned}
\Xi_{1}^{(\mathrm{wb})}= & \mathrm{i} \frac{g}{2 \omega_{1} V_{1}}\left(1-T_{1}^{2}\right)\left[k_{1}^{2} \mathscr{G}_{1}\left\{\widetilde{h}, \varphi_{2}\right\}+\mathrm{i} \varkappa_{1} P_{1} \mathscr{G}_{1}\left\{\widetilde{h}_{x}, \varphi_{2}\right\}\right. \\
& \left.+\mathrm{i} \lambda_{1}\left(q_{1} T_{1}-1\right) \mathscr{G}_{1}\left\{\widetilde{h}_{y}, \varphi_{2}\right\}\right], \\
\Xi_{1}^{(\mathrm{wbb})}= & \mathrm{i} \frac{g}{2 \omega_{1} V_{1}} k_{1}^{3} \frac{C_{1}}{2 C_{g, 1}} \frac{\left(1-T_{1}^{2}\right)^{2}}{T_{1}}\left[\frac{1}{\left(1-T^{2}\right)}-P_{1}\right] \mathscr{G}_{1}\left\{\widetilde{h}^{2}, \varphi_{2}\right\}, \\
\Xi_{1}^{(\mathrm{wc})}= & -\frac{1}{V_{1}}\left(\mathscr{G}_{1}\left\{\overline{\bar{\phi}}_{x}, \mathrm{i} \varkappa_{2} \varphi_{2}\right\}+\mathscr{G}_{1}\left\{\overline{\bar{\phi}}_{y}, \mathrm{i} \lambda_{2} \varphi_{2}\right\}\right),
\end{aligned}
$$

where

$$
P_{1}=\frac{1}{2}\left[\left(\frac{k_{1}}{\varkappa_{1}}\right)^{2}-1\right]-\frac{C_{1}}{2 C_{g, 1}}\left(1-q_{1} T_{1}\right)
$$

\section{Appendix C. Wave-driven mean flow and set-down}

Governing equations for the wave-driven mean flow are given to facilitate comparison to experimental (flume) data. Results are presented directly in physical variables and coordinates and we use the actual, two-dimensional depth, $h$, instead of the decomposition applied to derive the wave field evolution. The current-related quantities are assumed to depend on the slow scales in $x, y$ and $t$ :

$$
\left[\begin{array}{c}
\Phi^{(n, 0)} \\
\eta^{(n, 0)}
\end{array}\right]=\left[\begin{array}{c}
\Phi^{(n, 0)}\left(z, x_{1}, y_{1}, t_{1}, x_{2}, y_{2}, t_{2}\right) \\
\eta^{(n, 0)}\left(x_{1}, y_{1}, t_{1}, x_{2}, y_{2}, t_{2}\right)
\end{array}\right] .
$$

To the order of approximation required we obtain from the kinematic and dynamic free-surface boundary condition:

$$
\overline{\bar{\eta}}_{t}+\nabla \cdot(h \nabla \overline{\bar{\Phi}}+\boldsymbol{L})=0, \quad \overline{\bar{\eta}}=-\frac{1}{g} \overline{\bar{\Phi}}_{t}+B,
$$

where $\overline{\bar{\Phi}}=\epsilon \Phi^{(1,0)}$ and $\overline{\bar{\eta}}=\epsilon \eta^{(1,0)}+\epsilon^{2} / \mu \eta^{(2,0)}$. The wave-induced, depth-averaged mass flux $\boldsymbol{L}$ and the wave-induced water level correction $B$ are given by

$$
\begin{aligned}
\boldsymbol{L} & =\sum_{p_{1}} \frac{\omega_{1}}{g} \mathscr{F}^{-1}\left\{\varphi_{1} \mathrm{e}^{i \psi_{1}}\right\}\left\langle\mathscr{F}^{-1}\left\{\varkappa_{1} \varphi_{1} \mathrm{e}^{i \psi_{1}}\right\}^{*}, \mathscr{F}^{-1}\left\{\lambda_{1} \varphi_{1} \mathrm{e}^{i \psi_{1}}\right\}^{*}\right\rangle, \\
B & =\sum_{p_{1}} \frac{1}{2 g}\left[\left(k_{1} T_{1}\right)^{2}\left\|\mathscr{F}^{-1}\left\{\varphi_{1} \mathrm{e}^{i \psi_{1}}\right\}\right\|^{2}-\left\|\mathscr{F}^{-1}\left\{\mathrm{i} \varkappa_{1} \varphi_{1} \mathrm{e}^{i \psi_{1}}\right\}\right\|^{2}-\left\|\mathscr{F}^{-1}\left\{\mathrm{i} \lambda_{1} \varphi_{1} \mathrm{e}^{i \psi_{1}}\right\}\right\|^{2}\right],
\end{aligned}
$$

where $\varphi_{1}=\epsilon \varphi_{1}^{(1,1)}+(\epsilon / \mu)^{2} \varphi_{1}^{(2,2)}$. For a stationary wave field the depth-averaged, wavedriven mean flow and water level corrections are given by

$$
\nabla \cdot(h \nabla \overline{\bar{\Phi}}+\boldsymbol{L})=0, \quad \overline{\bar{\eta}}=B .
$$




\section{REFERENCES}

Agnon, Y., Madsen, P. A. \& Schäffer, H. A. 1999 A new approach to high-order Boussinesq models. J. Fluid Mech. 399, 319-333.

Agnon, Y. \& SHEREMET, A. 1997 Stochastic nonlinear shoaling of directional spectra. J. Fluid Mech. 345, 79-99.

Agnon, Y., Sheremet, A., Gonsalves, J. \& Stiassnie, M. 1993 Nonlinear evolution of a unidirectional shoaling wave field. Coast. Engng 20, 29-58.

Ardhuin, F. \& Herbers, T. H. C. 2002 Bragg scattering of random surface gravity waves by irregular sea bed topography. J. Fluid Mech. 451, 1-33.

Armstrong, J. A., Bloembergen, N., Ducuing, J. \& Pershan, P. S. 1962 Interactions between light waves in a nonlinear dielectric. Phys. Rev. 127, 1918-1939.

BEJI, S. 1995 Note on a nonlinearity parameter of surface waves. Coast. Engng 25, 81-85.

Beji, S. \& Battues, J. A. 1993 Experimental investigation of wave propagation over a bar. Coast. Engng 19, 151-162.

Beji, S. \& Battues, J. A. 1994 Numerical simulation of nonlinear wave propagation over a bar. Coast. Engng 23, 1-16.

Bredmose, H., Madsen, P. A., Schäffer, H. A. \& Agnon Y. 2002 Fully dispersive evolution equations: wave breaking and efficiency. Proc. 28th Intl Conf. Coastal Engng, Cardiff pp. 281-292. ASCE.

Bredmose, H., Schäffer, H. A., Madsen, P. A. \& Agnon Y. 2004 Boussinesq evolution equations: Numerical efficiency, breaking and amplitude dispersion. Coast. Engng 51, 1117-1142.

Bretherton, F. P. 1964 Resonant interactions between waves. The case of discrete oscillations. J. Fluid Mech. 20, 457-479.

Bryant, P. J. 1973 Periodic waves in shallow water. J. Fluid Mech. 59, 625-644.

BRYANT, P. J. 1974 Stability of periodic waves in shallow water. J. Fluid Mech. 66, 81-96.

BYrNE, R. J. 1969 Field occurences of induced multiple gravity waves. J. Geophys. Res. 74, 2590-2596.

Canuto, C., Hussaini, M. Y., Quarteroni, A. \& Zang, T. A. 1987 Spectral Methods in Fluid Dynamics. Springer.

Chu, V. H. \& MeI, C. C. 1970 On slowly varying Stokes waves. J. Fluid Mech. 41, 873-887.

Dalrymple, R. A. \& Kirby, J. T. 1988 Models for very wide-angle water waves and wave diffraction. J. Fluid Mech. 192, 33-50.

Dalrymple, R. A., Suh, K. D., Kirby, J. T. \& Chae, J. W. 1989 Models for very wide-angle water waves and wave diffraction. Part 2. Irregular bathymetry. J. Fluid Mech. 201, 299-322.

Davey, A. \& Stewartson, K. 1974 On three-dimensional packets of surface waves. Proc. R. Soc. Lond. A 338, 101-110.

Dingemans, M. W. 1997 Water Wave Propagation over Uneven Bottoms. World Scientific.

Dingemans, M. W., Petit, H. A. H., Meijer, Th. J. G. P. \& Kostense, J. K. 1991 Numerical evaluation of the third-order evolution equations for weakly nonlinear water waves propagating over uneven bottoms. In Comp. Modell. in Ocean Engng 91 (ed. A. S. Arcilla, M. Pastor, O. C. Zienkiewicz \& B. A. Schrefler), pp. 361-370. Balkema.

DJoRDJEvić, V. D. \& REDEKopP, L. G. 1978 On the development of packets of surface gravity waves moving over an uneven bottom. J. Appl. Maths Phys. 29, 950-962.

Dysthe, K. B. 1979 Note on a modification to the nonlinear Schrödinger equation for application to deep water waves. Proc. R. Soc. Lond. A 369, 105-114.

Eldeberky, Y. \& Madsen, P. A. 1999 Deterministic and stochastic evolution equations for fully dispersive and weakly non-linear waves. Coast. Engng 38, 1-24.

Elgar, S. \& GuZA, R. T. 1985 Observations of bispectra of shoaling surface gravity waves. J. Fluid Mech. 161, 425-448.

FeIR, J. E., 1967 Discussion: some results from wave pulse experiments. Proc. R. Soc. Lond. A 299, $54-58$.

Freilich, M. H. \& GuZA, R. T., 1984 Nonlinear effects on shoaling surface gravity waves. Proc. $R$. Soc. Lond. A 311, 1-41.

Hasimoto, H. \& Ono, H. 1972 Nonlinear modulation of gravity waves. J. Phys. Soc. Japan 33, 805-811.

Hasselmann, K. 1962 On the non-linear energy transfer in a gravity-wave spectrum. J. Fluid Mech. 49, 481-500. 
Herbers, T. H. C. \& Burton, M. C. 1997 Nonlinear shoaling of directionally spread waves on a beach. J. Geophys. Res. 102, 21101-21114.

Herterich, K. \& Hasselmann, K. 1980 A similarity relation for the nonlinear energy transfer in a finite-depth gravity-wave spectrum. J. Fluid Mech. 97, 215-224.

Hoefel, F. \& Elgar, S. 2003 Wave-induced sediment transport and sandbar migration. Science 299, $1885-1887$.

Hogan, S. J., Gruman, I. \& Stiassnie, M. 1988 On the changes in phase speed of one train of water waves in the presence of another. J. Fluid Mech. 192, 97-114.

Johnson, J. W., Fuchs, R. A. \& Morison, J. R. 1951 The damping action of submerged breakwaters. EOS Trans. AGU 32, 704-718.

Kaihatu, J. M. \& Kirby, J. T. 1995 Nonlinear transformation of waves in finite water depth. Phys. Fluids 7, 1903-1914.

Keller, J. B. 1988 Resonantly interacting water waves. J. Fluid Mech. 191, 529-534.

Kennedy, A. B. \& Kirby, J. T. 2003 An unsteady wave driver for narrowbanded waves: modeling nearshore circulation driven by wave groups. Coast. Engng 48, 257-275.

KIRBY, J. T. 1998 Discussion of 'Note on a nonlinearity parameter of surface waves' by S. Beji. Discussion in Coast. Engng 34, 163-168.

LAU, J. \& BARCILON, A. 1972 Harmonic generation of shallow water waves over topography. J. Phys. Oceanogr. 2, 405-410.

Lighthill, J. 1978 Waves in Fluids. Cambridge University Press.

LiU, P. L.-F. \& Dingemans, M. W. 1989 Derivation of the third-order evolution equations for weakly nonlinear water waves propagating over uneven bottoms. Wave Motion 11, 41-64.

LiU, P. L. -F., Yoon, S. B. \& Kirby, J. T. 1985 Nonlinear refraction-diffraction of waves in shallow water. J. Fluid Mech. 153, 185-201.

Lo, E. \& MeI, C. C. 1985 A numerical study of water-wave modulation based on a higher-order nonlinear Schrödinger equation. J. Fluid Mech. 150, 395-416.

Longuet-Higgins, M. S. \& Phillips, O. M. 1962 Phase velocity effects in tertiary wave interaction. J. Fluid Mech. 12, 333-336.

Luth, H. R., KLOPMAN, G. \& Kitou, N. 1994 Kinematics of waves breaking partially on an offshore bar; LDV measurements for waves with and without a net onshore current. Delft Hydraulics Rep. H1573. Delft Hydraulics, The Netherlands, 40pp.

Madsen, P. A., Bingham, H. B. \& Schäffer, H. A. 2003 Boussinesq-type formulations for fully nonlinear and extremely dispersive water waves: derivation and analysis. Proc. R. Soc. Lond. A 459, 1075-1104.

Madsen, P. A. \& SCHÄFfer, H. A. 1998 Higher-order boussinesq-type equations for surface gravity waves: derivation and analysis. Proc. R. Soc. Lond. A 356, 3123-3184.

Madsen, P. A. \& Sørensen, O. R. 1992 A new form of the Boussinesq equations with improved linear dispersion characteristics. Part 2: A slowly varying bathymetry. Coast. Engng 18, 183-204.

Madsen, P. A. \& Sørensen, O. R. 1993 Bound waves and triad interactions in shallow water. Ocean Engng 20, 359-388.

MeI, C. C. 1983 The Applied Dynamics of Ocean Surface Waves. J. Wiley \& Sons.

MeI, C. C. \& ÜnLÜATA, Ü. 1972 Harmonic generation in shallow water waves. In Waves on Beaches (ed. R. E. Meyer), pp. 181-202. Academic.

Munk, W. H. 1949 Surf beats. EOS Trans. AGU 30, 849-854.

Oppenheim, A. V. \& Schafer, R. W. 1989 Discrete-time Signal Processing. Prentice-Hall.

OrszaG, S. A. 1972 Comparison of pseudospectral and spectral approximations. Stud. Appl. Math 51, 253-259.

Peregrine, D. H. 1967 Long waves on a beach. J. Fluid Mech. 27, 815-827.

Peregrine, D. H. 1983 Water waves, nonlinear Schrödinger equations and their solutions. J. Austral. Math. Soc. B 25, 16-43.

Phillips, O. M. 1960 On the dynamics of unsteady gravity waves of finite amplitude, 1, the elementary interactions. J. Fluid Mech. 9, 193-217.

Phillips, O. M. 1977 The Dynamics of the Upper Ocean, 2nd Edn. Cambridge University Press.

RaYleigh, Lord 1876 On waves. Phil. Mag. 1 (4), 257-279.

Roelvink, J. A. \& Stive, M. J. F. 1989 Bar-generating cross-shore flow mechanisms on a beach. J. Geophys. Res. 94, 4785-4800. 
Shemer, L., Jiao, H., Kit, E. \& Agnon, Y. 2001 Evolution of a nonlinear wave field along a tank: experiments and numerical simulations based on the spatial Zakharov equation. J. Fluid Mech. 427, 107-129.

Shemer, L., Kit, E., JiaO, H. \& Eitan, O. 1998 Experiments on nonlinear wave groups in intermediate water depth. J. Waterway, Port, Coast. Ocean Engng 124, 320-327.

Sheremet, A. 1996 Wave interaction in shallow water. ScD thesis, Technion, Haifa, Israel, 100 pp.

Stamnes, J. J. 1986 Waves in Focal Regions. Adam Hilger.

Stiassnie, M. \& SHemer, L. 1984 On modifications of the Zakharov equation for surface gravity waves. J. Fluid Mech. 143, 47-67.

Stokes, G. G. 1847 On the theory of oscillatory waves. Trans. Camb. Phils. Soc. 8, 441-455.

Stokes, G. G. 1880 Supplement to a paper on the theory of oscillatory waves. Math. Phys. Papers vol. 1, pp. 314-326. Cambridge University Press.

Suh, K. D., Dalrymple, R. A. \& Kirby, J. T. 1990 An angular spectrum model for propagation of Stokes waves. J. Fluid Mech. 221, 205-232.

TANG, Y. \& Ouellet, Y. 1997 A new kind of nonlinear mild-slope equation for combined refractiondiffraction of multifrequency waves. Coast. Engng 31, 3-36.

Westhuis, J. 2001 The numerical simulations of nonlinear waves in a hydrodynamic model test basin. PhD thesis, Twente University of Technology, Netherlands, $255 \mathrm{pp}$.

WhaLIN, R. W. 1971 The limit of application of linear wave refraction theory in a convergence zone. Res. Rep. H-71-3. US Army Engineer Waterways Experiment Station, Vicksburg (MS), $156 \mathrm{pp}$.

WiLlebrand, J. 1973 Zum Energietransport in einem nichtlinearen räumlich inhomogenen Seegangsfeld. PhD thesis, Der Christian-Albrechts-Universität, Kiel, Germany, 55 pp.

WiLlebrand, J. 1975 Energy transport in a nonlinear and inhomogeneous random gravity wave field. J. Fluid Mech. 70, 113-126.

Yuen, H. C. \& LaKe, B. M. 1975 Nonlinear deep water waves: Theory and experiment. Phys. Fluids 18, 956-960.

ZaKharov, V. E. 1968 Stability of periodic waves of finite amplitude on the surface of a deep fluid. Trans. ASME: J. Appl. Mech. 4, 86-94. 